\title{
IL VOTO PROPORZIONALE E IL NUOVO SPAZIO POLITICO ITALIANO
}

\author{
Di Luca Ricolfi
}

Questo saggio si occupa esclusivamente del voto di lista (ai singoli partiti), espresso mediante la «scheda proporzionale» della Camera alle elezioni politiche del 27 e 28 marzo 1994. Il suo scopo è essenzialmente di ritracciare la mappa politica dell'Italia, sia in termini territoriali ${ }^{1}$ sia in termini di geometria dello spazio elettorale ${ }^{2}$. Quest'ultimo aspetto verrà affrontato, in questo lavoro, esclusivamente sotto il profilo macro, considerando come unità di analisi i collegi o le circoscrizioni e non gli individui.

Al momento in cui scrivo (luglio 1994) si sono già svolte le elezioni Europee, nonché le amministrative in Sardegna e in molti comuni. In queste due circostanze l'elettorato ha notevolmente modificato le proprie scelte precedenti: in occasione del voto europeo premiando ulteriormente Forza Italia, in occasione del voto amministrativo concedendo alle opposizioni di centro e di sinistra una parziale rivincita.

$\mathrm{Ci}$ si potrebbe chiedere, quindi, fino a che punto un'analisi del voto di marzo non sia già superata dagli esiti del voto di giugno. La mia opinione è che non lo sia affatto, perché i due «segnali» del voto europeo e del voto amministrativo viaggiano su lunghezze d'onda diverse rispetto a quella del voto politico.

Sono grato ad Alfio Mastropaolo e Rocco Sciarrone per le loro osservazioni alla prima stesura di questo lavoro.

1 Per un quadro delle varie proposte di partizione dell'Italia in zone geopolitiche vedi Cartocci (1987), Ricolfi (1989, 1993b), Sani (1993a, 1993b, 1994), Natale (1994).

2 Per spazio elettorale intendiamo qui uno spazio metrico i cui punti sono costituiti esclusivamente da liste elettorali e/o segmenti di elettorato, e non da «stimoli» più o meno connotati (l'Unione sovietica, il sindacato, lo stato, i magistrati etc.). Fra gli studi recenti sulla geometria dello spazio elettorale in Italia ricordiamo Mannheimer, Sani (1987), Corbetta, Parisi, Schadee (1988), Corbetta (1993), Ricolfi (1993b, 1994a, 1994b).

RIVISTA ITALIANA DI SCIENZA POLITICA / a. XXIV, n. 3, dicembre 1994 
Non solo, ma una lettura del voto eccessivamente focalizzata su quel che è successo «dopo» rischia di essere altamente fuorviante, specie per i vincitori.

La rivincita del centro in Sardegna è in gran parte connessa al carisma di Segni, e al conseguente radicamento del Patto nell'isola. È difficile pensare che ad essa corrisponda un'inversione di tendenza per l'elettorato nel suo insieme.

$\mathrm{Ma}$ anche lo sfondamento di Forza Italia alle Europee va preso con una certa cautela, sia per il carattere particolare di queste elezioni (ricordate l'improvviso successo del Pci nel 1984, subito dopo la morte di Berlinguer?), sia perché lo spostamento di consensi intervenuto fra i due tipi di consultazione difficilmente può essere ascritto a un cambiamento repentino e radicale degli orientamenti politici di fondo dell'elettorato, i soli che ci interessano in questa sede. Nel trionfo della destra alle europee ritroviamo, semmai, una sorta di mix dei più classici meccanismi della psicologia del consumatore: effetto di dimostrazione (Duesenberry 1949), band wagon (Leibenstein 1950), formazione adattiva delle preferenze (Elster 1983) ${ }^{3}$. Tutto lascia supporre che una vittoria della sinistra alle politiche avrebbe prodotto uno spostamento di entità analoga e di segno opposto.

La geografia del voto europeo è ampiamente «drogata» da tre fattori: il basso tasso di partecipazione al voto; la naturale tendenza dell'elettore a ridurre la dissonanza cognitiva, saltando sul carro del vincitore; l'amplificazione di questa tendenza nel Mezzogiorno, che percepisce i partiti in modo radicalmente diverso dal resto del paese ${ }^{4}$.

3 Per una rassegna dei meccanismi della psicologia del consumatore vedi Amaturo e Ragone (1979). Sulla prevalenza dei meccanismi di formazione adattiva rispetto a quelli di formazione contro-adattiva delle preferenze vedi Ricolfi (1990).

4 Un'analisi della percezione dello spazio elettorale condotta separatamente nelle principali zone geopolitiche del paese mostra:

a) che l'elettorato del Mezzogiorno percepisce i partiti in modo unidimensionale, anziché bidimensionale come gli elettorati delle altre zone;

b) che la dimensione lungo cui gli elettori meridionali percepiscono i partiti è interpretabile come il prodotto fra l'entità della propensione redistributiva e le probabilità di accesso al governo di ciascuno di essi (una sorta di «utilità attesa» associata ad ogni partito).

Questa struttura delle preferenze ha una precisa implicazione empirica. Se l'esito dello scontro è incerto, le probabilità di accesso al governo rifletteranno le esperienze passate (Msi probabilità minime, Dc probabilità massime), ma se una consultazione elettorale precedente (politiche 1994) modifica le valutazioni iniziali a favore di determinati partiti (Forza Italia e Msi nella fattispecie), allora le rispettive utilità attese sono destinate a salire, e con esse il consenso ad essi accordato dall'elettorato meridionale. 
Ecco perché, se si vogliono capire le differenze e le tendenze profonde che il voto di primavera ha espresso, è più saggio soffermarsi sul risultato delle politiche.

\section{Verso il 27 marzo}

Per cogliere il senso di quel che è successo alle politiche del 1994 occorre avere dei termini di riferimento. Il termine di riferimento più naturale è dato dalle politiche precedenti, tenutesi appena due anni prima (5 aprile del 1992). Ma in questo caso due anni sono un tempo lunghissimo, che non permette di cogliere la complessità dei processi che sono intervenuti fra le due consultazioni. Consideriamo, per fare l'esempio più clamoroso, il caso della Lega Nord. Alle politiche del 1992 aveva l'8,7\% dei consensi, alle politiche del 1994 aveva l'8,4\%. Dunque non è successo nulla?

Allora diamo un'occhiata ai sondaggis condotti fra la primavera e l'estate del 1993. In quel periodo i consensi alla Lega erano prossimi al $20 \%$. Rispetto a quest'ultimo termine di confronto il risultato del 27 marzo rappresenta una vera débâcle: la Lega è più che dimezzata in pochi mesi.

Per apprezzare meglio l'entità del cambiamento intervenuto fra il 1992 e il 1994 abbiamo affiancato ai risultati ufficiali delle elezioni politiche quelli di alcuni sondaggi ${ }^{6}$ condotti dall'Istituto Cirm fra l'aprile del 1993 e il febbraio del 1994 (Tab. 1). I valori riportati possono essere confrontati fra loro, ma vanno usati

Ecco perché, conformemente alle predizioni del modello, l'effetto di trascinamento fra elezioni politiche ed europee è stato più forte nel Mezzogiorno che nel resto del paese.

L'analisi è stata condotta con la tecnica dello scaling multidimensionale su dati di preferenza (ordinamenti completi dei partiti) di fonte Cra (Consulenti ricercatori associati). I risultati sono stati presentati, prima delle politiche del 1994, a un seminario sulla Lega tenutosi presso l'Istituto di Studi Orientali dell'Università di Napoli il 17 marzo 1994.

5 Una rassegna dei principali sondaggi condotti dagli Istituti demoscopici fra il marzo del ' 93 e il gennaio del '94 è stata presentata da Ezio Marra a un seminario sulle tendenze del voto tenutosi presso la Fondazione Gramsci di Torino il 10 febbraio del 1994. Per quanto riguarda $i$ dati ufficiali del 1994 qui e in tutte le analisi successive $i$ Verdi includono anche i Verdi-Verdi, Forza Italia include anche il Ccd (Centro cristiano democratico), Ad include anche la lista Ada.

${ }^{6}$ I sondaggi dell'Istituto Cirm considerati sono cinque. Le interviste dei primi tre sono state effettuate nei mesi di aprile, settembre e ottobre del 1993, quelle degli altri due sono state effettuate il 4 e il 10 febbraio del 1994. Nella tabella 1 la colonna relativa al mese di febbraio contiene i risultati medi di questi ultimi due sondaggi. 
TAB. 1. Evoluzione delle preferenze elettorali nella XI legislatura

\begin{tabular}{|c|c|c|c|c|c|c|c|}
\hline & Voto & & Son & daggi & & Voto & \\
\hline $\begin{array}{l}\text { Vecchi } \\
\text { simboli }\end{array}$ & $\begin{array}{c}1992 \\
\text { aprile }\end{array}$ & $\begin{array}{c}1993 \\
\text { aprile }\end{array}$ & $\begin{array}{r}19 \\
\text { sett }\end{array}$ & 93 ott & $\begin{array}{c}1994 \\
\text { inizio feb }\end{array}$ & $\begin{array}{c}1994 \\
\text { marzo }\end{array}$ & $\begin{array}{l}\text { Nuovi } \\
\text { simboli }\end{array}$ \\
\hline $\begin{array}{l}\text { Rc } \\
\text { Pds } \\
\text { Rete } \\
\text { Verdi } \\
\text { Psi }\end{array}$ & $\begin{array}{c}5,6 \\
16,1 \\
1,9 \\
2,8 \\
13,6\end{array}$ & $\begin{array}{r}7 \\
17 \\
3 \\
2 \\
7 \\
8\end{array}$ & $\begin{array}{r}5 \\
18 \\
3 \\
4 \\
5 \\
5\end{array}$ & $\begin{array}{r}7 \\
19 \\
5 \\
4 \\
4 \\
3\end{array}$ & $\begin{array}{r}4,5 \\
20,0 \\
2,0 \\
3,0 \\
1,5 \\
2,0\end{array}$ & $\begin{array}{r}6,0 \\
20,4 \\
1,9 \\
2,7 \\
2,2 \\
1,2\end{array}$ & $\begin{array}{l}\text { Rc } \\
\text { Pds } \\
\text { Rete } \\
\text { Verdi } \\
\text { Psi } \\
\text { Ad }\end{array}$ \\
\hline PROGR. & 40,0 & 44 & 40 & 42 & 33,0 & 34,4 & PROGR. \\
\hline Pri & 29,7 & 20 & 20 & 19 & $\begin{array}{r}0,5 \\
8,0 \\
10,0\end{array}$ & $\begin{array}{r}4,7 \\
11,1\end{array}$ & $\begin{array}{l}\text { Patto } \\
\text { Ppi }\end{array}$ \\
\hline CENTRO & 34,1 & 23 & 23 & 21 & 18,5 & 15,8 & CENTRO \\
\hline Pann & 1,2 & 2 & 4 & 3 & 1,0 & 3,5 & Pann \\
\hline $\begin{array}{l}\text { Psdi } \\
\text { Pli }\end{array}$ & $\begin{array}{l}2,7 \\
2,8\end{array}$ & $\begin{array}{l}3 \\
2\end{array}$ & $\begin{array}{l}2 \\
2\end{array}$ & $\begin{array}{l}1 \\
1\end{array}$ & $\begin{array}{r}0,5 \\
0,5 \\
21,5\end{array}$ & 21,0 & $\mathrm{Fi}$ \\
\hline $\begin{array}{l}\text { Lega } \\
\text { Msi }\end{array}$ & $\begin{array}{l}8,7 \\
5,4\end{array}$ & $\begin{array}{r}19 \\
8\end{array}$ & $\begin{array}{r}19 \\
7\end{array}$ & $\begin{array}{r}21 \\
8\end{array}$ & $\begin{array}{l}11,0 \\
10,5\end{array}$ & $\begin{array}{r}8,4 \\
13,5\end{array}$ & $\begin{array}{l}\text { Lega } \\
\text { An }\end{array}$ \\
\hline DESTRA & 19,6 & 32 & 30 & 31 & 44,0 & 42,9 & DESTRA \\
\hline Altri & 5,1 & 0 & 3 & 3 & 3,5 & 3,4 & Altri \\
\hline Totale & 100 & 100 & 100 & 100 & 100 & 100 & Totale \\
\hline
\end{tabular}

con prudenza nei raffronti con i risultati ufficiali; tutto lascia pensare, infatti, che essi tendano a sovrastimare leggermente la destra, in parte a danno del centro, in parte a danno della sinistra $^{7}$. Nel commento che segue ce ne serviremo solo per cogliere le tendenze più importanti, e inoltre terremo conto, nel valutare la forza dei tre schieramenti, della piccola distorsione di cui si è detto.

La tabella mostra quanti cambiamenti siano intervenuti nel breve intervallo di due anni. La forza principale del vecchio si-

7 Siamo giunti a questa conclusione confrontando i dati Cirm sia con i risultati effettivi del 27 marzo, sia con le stime della forza dei vari schieramenti desumibili da un uso congiunto dei risultati ufficiali e del panel $\mathrm{Cra}$. Tali confronti suggeriscono che l'ordine di grandezza della sovrastima della destra da parte del Cirm sia di 2-4 punti. 
stema politico, la Dc, già un anno dopo il voto del '92 aveva perso 10 punti, ossia un terzo del suo elettorato. Dopo quasi un anno di tregua, in cui il declino sembra essersi fermato sulla soglia del $20 \%$, altri $4-5$ punti li perderanno i suoi eredi - Partito popolare e Patto Segni - nei due mesi che precedono il 27 marzo, probabilmente anche grazie all'iniquo trattamento riservato alle forze di centro da parte dell'informazione televisiva ${ }^{8}$. Al declino della Dc si accompagna il crollo dei consensi al Psi, che in un anno dimezza il suo elettorato (dal 14\% al $7 \%$ ), per poi perderlo quasi interamente nel corso dell'anno seguente (dal $7 \%$ al $2 \%)$.

A fronte di questa crisi delle due principali forze di governo fa riscontro prima un aumento simmetrico e speculare delle opposizioni di sinistra e di destra, poi - a partire dall'estate del 1993 - un declino dei consensi alla sinistra e un'ulteriore espansione della destra.

Nel primo anno l'espansione della sinistra avviene attraverso tre canali fondamentali: il rafforzamento degli eredi del Pci (Rifondazione comunista e Pds), la crescita dei consensi alle due forze laiche minori dello schieramento di sinistra (Verdi e Rete), ma soprattutto la nascita e l'ascesa di Alleanza democratica. Nel sondaggio condotto dal Cirm nell'aprile del 1993, ossia esattamente a metà della XI Legislatura, Alleanza democratica tocca l' $8 \%$ dei consensi assurgendo così al rango di quarta forza politica del paese (dopo Dc, Lega e Pds, e alla pari con il Msi).

Sul versante della destra il grosso della crescita è dovuto alla Lega, che nel giro di soli 12 mesi raddoppia i propri consensi passando dall' $8,7 \%$ a quasi il $20 \%$.

Complessivamente nel primo anno di legislatura la sinistra (con Ad, ma senza Psi, Pri e Pannella) passa dal 26,4\% al 37 $39 \%$ dei consensi, la destra (Msi e Lega) passa dal $14,1 \%$ al 25 $27 \%$. Questo significa che, in una prima fase, il «bottino» del

8 Tutte le ricerche sul comportamento della televisione nel periodo precedente le elezioni hanno rilevato la penalizzazione del centro. $\mathrm{Ci}$ riferiamo, in particolare, alle analisi dei tempi e dei contenuti delle trasmissioni effettuate dall'Osservatorio di Pavia (diretto da Franco Rositi, Giacomo Sani e Pasquale Scaramozzino) e dal seminario Mediamonitor (coordinato da Mario Morcellini), nonché alle stime, da me ottenute in base a dati Cra, dell'impatto che l'esposizione alle reti Rai e Fininvest ha avuto sul voto di marzo. Al momento in cui scrivo i risultati di queste tre ricerche, più volte presentati in seminari e convegni tenutisi nei mesi successivi al voto, non sono ancora stati pubblicati in sede scientifica. 
quadripartito viene spartito pressapoco alla pari fra sinistra e destra. A questa spartizione danno un contributo decisivo Alleanza democratica su un versante e la Lega sull'altro: il $75 \%$ dell'espansione della sinistra confluisce su Ad, l'80\% dell'espansione della destra confluisce sulla Lega.

Ma questa situazione è destinata a cambiare radicalmente a partire dall'estate. Sul versante progressista la crisi di Ad, che nel giro di 6 mesi scende dall' $8 \%$ al $3 \%$ dei consensi, dapprima provoca una lievitazione degli altri partiti del fronte progressista (e segnatamente della Rete e dei Verdi), poi, non appena Segni esce da Ad e comincia a delinearsi l'ingresso in campo di Berlusconi, finisce per tradursi in un indebolimento dell'intero fronte progressista. $\grave{E}$ difficile dire quando, esattamente, inizi questo esaurimento della capacità della sinistra di intercettare i voti «in uscita» dal quadripartito. A giudicare dai molti sondaggi condotti fra la primavera del 1993 e la primavera del 1994 l'inversione nel trend della sinistra sembra situarsi intorno al mese di settembre del 1993, ossia in prossimità dell'uscita di Segni da Alleanza democratica. A partire da questo momento sia l'elettorato di centro sia una parte considerevole dell'elettorato temporaneamente «catturato» dal fronte progressista cominciano a guardare altrove. E molto probabile che, durante la euforica campagna d'autunno per l'elezione dei sindaci delle grandi città, il fronte progressista fosse già in declino, un declino che la nascita di Forza Italia e la decisione di Berlusconi di entrare in politica non faranno che accelerare. A cavallo di $\mathrm{Na}$ tale, nei quattro mesi che vanno dall'ottobre del 1993 al febbraio del 1994, il consenso alla sinistra si riduce in una misura che è difficile quantificare con precisione, ma che può essere ragionevolmente valutata intorno al $15-20 \%$ del proprio elettorato iniziale. Collocata stabilmente al di sopra del $40 \%$ nella maggior parte dei sondaggi fino ad ottobre ' 93 , la sinistra precipita appena al di sopra del $35 \%$ tra la fine di gennaio e l'inizio di febbraio del '94.

Parallelamente, sull'altro versante dello scacchiere politico, la nascita di Forza Italia consente un'ulteriore rafforzamento della destra, che si porta dal $30 \%$ circa di ottobre al $38-40 \%$ dell'inizio di febbraio. Ma questo processo non avviene attraverso una crescita equilibrata di tutte le sue componenti bensì attraverso una sorta di «cannibalizzazione» degli alleati da parte del partito di Berlusconi. A parte i liberali (Unione di centro) e gli ex democristiani (Ccd), direttamente assorbiti (e salvati da 
estinzione sicura) sotto le insegne di Forza Italia, sono soprattutto i leghisti e gli ex missini di Alleanza nazionale a fare le spese dell'irresistibile ascesa del Cavaliere. La Lega in modo diretto, attraverso il completo riassorbimento dei nuovi consensi ottenuti dopo le politiche del 1992. Alleanza nazionale in modo strisciante, attraverso la sterilizzazione del suo elettorato potenziale. Una serie di indagini Cra condotte su un panel nazionale fra la primavera del 1993 e la primavera del 1994 mostra che circa un quarto dell'elettorato di Forza Italia considera il Movimento Sociale come la propria seconda scelta, ossia come il partito preferito subito dopo il proprio. Un po' come Berlusconi stesso (che durante la campagna per l'elezione del sindaco di Roma aveva dichiarato: «meglio Fini che Rutelli»), questi elettori - senza essere missini - erano, per così dire, pronti a diventarlo in caso di necessità.

In questo medesimo periodo, in cui la sinistra perde colpi e la destra aumenta i suoi consensi, la forza del centro subisce un ulteriore ridimensionamento, passando dal $22-23 \%$ di settembre-ottobre 1993 al $18-20 \%$ dell'inizio di febbraio'. È verosimile che questo ridimensionamento sia la risultante di due forze di segno opposto: un'emorragia di consensi verso la destra, e un recupero di consensi da sinistra. La crisi della sinistra, come vedremo meglio nel prossimo paragrafo, ha infatti due effetti distinti: il primo è di alimentare, attraverso i passaggi diretti, la crescita della destra, il secondo è di frenare il declino del centro.

\section{La volata finale}

Se le linee generali del cambiamento fra le politiche del ' 92 e l'inizio di febbraio del '94 sono relativamente chiare, molto meno nitidi sono i processi intervenuti nelle ultime 6-7 settimane prima del voto. Analizzando e confrontando le risultanze dei sondaggi Cirm e Cra, gli unici condotti con sufficiente continui-

9 Le valutazioni che compaiono nel testo sulla forza rispettiva della sinistra (35\%), del centro $(18-20 \%)$ e della destra $(38-40 \%)$ all'inizio di febbraio poggiano su una nostra ricostruzione (mediante dati $\mathrm{Cra}$ ) degli orientamenti elettorali all'inizio di febbraio. Tale ricostruzione è basata sull'uso combinato dei risultati effettivi (ufficiali) del 27 marzo e delle matrici di transizione ricavabili dal panel Cra. Rispetto alle valutazioni del Cirm (Tab. 1) le nostre stime risultano leggermente più favorevoli al centro e alla sinistra, e meno favorevoli alla destra. 
TAB. 2. Matrice di flusso dell'ultima settimana di campagna elettorale

\begin{tabular}{lrrrrr}
\hline & \multicolumn{5}{c}{20 Marzo } \\
27 Marzo & Sin & Cen. & Des. & Pann. & Voto \\
\hline Sinistra & 78,0 & 8,6 & 3,7 & 19,4 & 34,4 \\
Centro & 4,7 & 64,6 & 6,0 & 0,0 & 15,8 \\
Destra & 11,2 & 21,1 & 84,1 & 9,7 & 42,9 \\
Pannella & 1,6 & 3,8 & 3,3 & 64,5 & 3,5 \\
Altri & 4,5 & 1,9 & 2,9 & 6,4 & 3,4 \\
& 100,0 & 100,0 & 100,0 & 100,0 & 100,0 \\
\hline Orientamento di & & & & & \\
voto al 20 Marzo & $(39,9)$ & $(17,8)$ & $(41,0)$ & $(1,3)$ &
\end{tabular}

tà durante i due mesi anteriori al voto, sembra possibile fissare questi sei punti:

a) l'ultima, significativa, onda di espansione della destra, trainata dall'ingresso di Forza Italia nell'arena politica, è sostanzialmente esaurita con la prima decade di febbraio;

b) l'esaurimento dell'onda lunga della destra interrompe, o comunque frena, il declino del centro;

c) più o meno nello stesso periodo ${ }^{10}$ si interrompe anche il declino della sinistra, iniziato nell'autunno del 1993;

d) nella prima decade di febbraio la destra sfiora il $40 \%$, il centro il $20 \%$, e la sinistra è appena al di sopra del $35 \%$;

$e$ ) in tutto il mese successivo, dalla metà di febbraio alla metà di marzo, i rapporti di forza fra i tre schieramenti - destra, sinistra e centro - non subiscono modificazioni di grande entità; se un trend si può rintracciare nei dati esso va nel senso di un certo consolidamento della sinistra (da 2 a 4 punti), soprattutto a spese del centro;

f) lo spostamento cruciale nei rapporti di forza avviene nell'ultima settimana della campagna elettorale: fra il 20 e il 27 marzo la sinistra perde quasi 4 punti, il centro ne perde quasi 2 , e il bottino viene ripartito fra le forze del cartello di destra e la lista Pannella.

$\mathrm{Ma}$, con l'aiuto del panel $\mathrm{Cra}$, andiamo a vedere meglio la «microfisica» della decisione di voto, come si è arrivati - nel-

${ }^{10}$ Nella prima decade di febbraio in base alla serie Cra (indagini quindicinali, dal 6 febbraio al 20 marzo), nella seconda in base alla serie Cirm (indagini settimanali, dal 21 gennaio al 10 marzo). 
l'ultima settimana - a capovolgere una situazione che appariva di sostanziale equilibrio. Utilizzando la matrice di flusso che collega gli orientamenti della prima decade di marzo con le scelte effettive di voto del 27 e 28 marzo si ottiene il quadro illustrato dalla tabella 2.

Poiché gli Altri (liste locali tradizionali, come la Südtiroler Volkspartei, e liste «fai da te») non erano presenti fra le alternative sottoposte alla scelta una settimana prima del voto, per ricostruire l'evoluzione dei rapporti di forza nell'ultima settimana di campagna elettorale occorre - anche il 20 marzo - sottrarre ai vari schieramenti quel $3,4 \%$ di consensi che una settimana dopo è confluito nelle «Altre liste». Compiendo questa operazione in funzione delle informazioni contenute nella matrice di flusso si perviene a questi risultati:

ТАВ. 3. Variazioni fra il 20 e il 27 marzo

\begin{tabular}{lccc}
\hline & 20 Marzo & 27 Marzo & Variaz. \\
\hline Sinistra & 38,1 & 34,4 & $-3,7$ \\
Centro & 17,5 & 15,8 & $-1,7$ \\
Destra & 39,8 & 42,9 & 3,1 \\
Pannella & 1,4 & 3,5 & 2,3 \\
Altri & 3,4 & 3,4 & 0,0 \\
\hline
\end{tabular}

L'esercizio è interessante perché mostra che, comunque la correzione venga effettuata, il risultato è un quadro di sostanziale equilibrio fra sinistra e destra ad appena una settimana dal voto $(38,1$ contro 39,8$)$.

Vediamo dunque che cosa, nelle ultime tre settimane, ha modificato questo equilibrio iniziale. La tabella 2 mostra, innanzitutto, la grande fluidità dell'elettorato. Su 100 elettori che avevano espresso un certo orientamento il 20 marzo, solo 80 lo hanno confermato una settimana dopo ${ }^{11}$. Ma gli elettori «infedeli» non si sono comportati in modo erratico: $i$ capovolgimenti di fronte (da sinistra a destra e viceversa) sono prevalsi nettamente sugli spostamenti verso il centro e, nell'ambito degli spostamenti del primo tipo, quelli da sinistra a destra sono stati molto più numerosi di quelli in direzione opposta. Nel giro di una sola settimana la sinistra ha ceduto agli altri schieramenti liste.

1 I calcoli dei tassi di fedeltà e di «tradimento» sono effettuati al netto delle Altre 
circa 22 elettori su 100, mentre è riuscita ad attirarne nella propria orbita solo 8. La destra, viceversa, ha ceduto quasi 16 elettori su 100 ma ne ha attirati oltre 20 .

E il caso di sottolineare che questa struttura dei flussi, basata su una circolazione che - soprattutto da sinistra a destra tende a «saltare» il centro, non può essere spiegata con le caratteristiche del sistema elettorale, ed è incompatibile con gli assunti base del modello di Black-Coombs (unidimensionalità). Il bipolarismo, che per alcuni sarebbe un portato del nuovo sistema elettorale, può essere invocato per spiegare il prosciugamento del centro (gli elettori di centro non vogliono rischiare che il proprio voto sia utilizzato dal polo «sbagliato»), ma non per rendere conto della entità dei passaggi diretti fra le estreme. Questa può essere spiegata in modo limpido solo abbandonando l'assunto di single-peakedness delle funzioni di utilità individuali, ossia assumendo la non unidimensionalità dello spazio sottostante ai movimenti elettorali.

Anche senza invocare gli studi che si sono occupati di ricostruire in modo esplicito la geometria dello spazio elettorale ${ }^{12}$, il carattere non unidimensionale dello spazio che governa i flussi elettorali può essere colto direttamente calcolando - con l'aiuto di un modello gravitazionale ${ }^{13}-$ le distanze fra gli schieramenti implicite nella matrice di flusso.

Questa operazione può essere condotta separatamente sulle due metà della matrice, in modo da evidenziare le differenze fra i due regimi di circolazione degli elettori. Il modo più semplice di ottenere le distanze reciproche fra sinistra, centro e destra è di utilizzare direttamente la formula della gravitazione universale di Newton, secondo cui la forza di attrazione che due corpi esercitano fra di loro è direttamente proporzionale alle rispettive masse $\left(\mathrm{m}_{i}\right.$ e $\left.\mathrm{m}_{i}\right)$, e inversamente proporzionale al quadrato della loro distanza $\left(\mathrm{d}_{i j}\right)$ :

$$
\mathrm{a}_{i j}=\mathrm{m}_{i} \mathrm{~m}_{j} / \mathrm{d}_{i j}^{2}
$$

ovvero:

$$
\mathrm{d}_{i j}=\left(\mathrm{m}_{i} \mathrm{~m}_{j} / \mathrm{a}_{i j}\right)^{1 / 2}
$$

${ }_{12}$ Mi riferisco all'uso di tecniche di scaling su dati di preferenza di tipo individuale. Vedi Ricolfi (1994a e 1994b).

${ }_{13}$ Sulla logica dei modelli gravitazionali si veda, ad esempio, Hagger et al. (1977), sul loro impiego nell'analisi delle tavole di mobilità Bianco, Ricolfi (1993). 
Per usare la formula basta sostituire alle masse le percentuali marginali, e all'attrazione reciproca le percentuali di cella ricavabili dalla tabella 2.

$\mathrm{m}_{i} \rightarrow$ percentuale dello schieramento $i$

$\mathrm{m}_{j} \rightarrow$ percentuale dello schieramento $j$

$\mathrm{a}_{i j} \rightarrow$ percentuale di casi nella cella $i j$

È facile rendersi conto che la distanza risultante non è altro che il reciproco di un ben noto coefficiente usato nella letteratura sulla mobilità sociale: il social distance mobility ratio (Rogoff 1953), o index of association (Glass 1954).

I risultati ottenuti sono rappresentati nelle figure $1 \mathrm{a}$ e $1 \mathrm{~b}$.

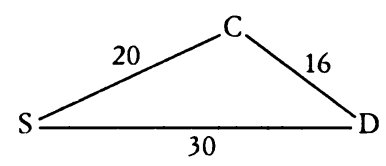

(a) circolazione a sinistra

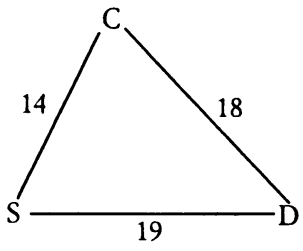

(b) circolazione a destra

FIG. 1. Metrica sottostante alla matrice di flusso

Le due figure mostrano che in entrambi i casi il centro non sta fra la sinistra e la destra ma sta sopra l'asse che congiunge l'una all'altra. Nel caso degli elettori che si sono spostati verso sinistra questo «stare sopra» del centro non è particolarmente pronunciato, e il triangolo ottusangolo che ne risulta può forse ancora, con qualche forzatura, essere ricondotto al caso di unidimensionalità («unidimensionalità imperfetta» $\left.{ }^{14}\right)$ : se immaginiamo di abbassare ulteriormente il vertice che rappresenta il centro possiamo immaginare di trasformare il nostro triangolo, che è una figura piana, in un segmento con tre punti, ossia in

14 Il concetto di «unidimensionalità imperfetta» allude all'impiego di modelli che, pur essendo sostanzialmente unidimensionali, incorporano uno o più parametri per tenere conto di specifiche anomalie (ad esempio la presenza di un partito cattolico). Sulla distinzione fra unidimensionalità imperfetta e multidimensionalità si veda Ricolfi (1993b). Per il caso italiano, un buon esempio di unidimensionalità imperfetta è quello che emerge dagli studi dell'Istituto Cattaneo anteriori alla comparsa della Lega sulla scena politica (Corbetta, Parisi e Schadee 1988). 
una figura di dimensione uno. Nel caso degli elettori che si sono spostati verso destra questa operazione di «schiacciamento» diventa del tutto improponibile. Il triangolo che descrive la logica dei loro movimenti è un triangolo con tutti gli angoli acuti, molto simile a un triangolo equilatero. La seconda dimensione non può essere soppressa, ma va interpretata ${ }^{15}$.

\section{La nuova mappa del voto e la «quarta Italia»}

Soffermiamoci ora sull'esito finale degli spostamenti che abbiamo provato a ricostruire. Rispetto alla situazione che si era determinata all'indomani del 5 aprile 1992 la mappa del voto appare decisamente cambiata.

Allora la maggioranza di quadripartito aveva tenuto solo nelle regioni meridionali, mentre nel centro-nord avevano prevalso le opposizioni (Lega e Pds innanzitutto). Il governo centrale, ancora fondato sull'alleanza di quadripartito (governo Amato), era la proiezione delle preferenze elettorali delle regioni del Mezzogiorno.

L'Italia appariva nettamente divisa in tre aree: il Nord leghista, il Centro comunista e il Sud governativo (Ricolfi 1993b). Anche chi, come Giacomo Sani, preferiva distinguere fra due Sud, e parlare quindi di quattro Italie, in realtà si limitava a suddividere le province meridionali soprattutto sulla base di una variabile - la tenuta della Dc - che permetteva di individuare importanti differenze di grado, ma non di isolare due realtà territoriali nitidamente distinte sul piano della struttura dei consensi (Sani 1993a, 1993b, 1994).

Come si vede dalla tabella 4, le differenze reciproche fra $\mathrm{Pa}$ dania, Etruria e Mezzogiorno sono molto più pronunciate di quelle fra Sud-1 (prevalentemente costiero) e Sud-2 (prevalentemente interno).

Due anni dopo la situazione è drasticamente cambiata. La Dc non c'è più, e i suoi eredi (Partito popolare e Patto Segni) si

15 Se ci collochiamo in una prospettiva micro, e ci chiediamo quindi come gli individui percepiscono lo spazio elettorale, possiamo tentare un'analisi del significato della seconda dimensione che governa i flussi fra schieramenti. Da un'analisi di questo genere, condotta sia con tecniche di scaling sia con tecniche fattoriali, emerge piuttosto chiaramente che la seconda dimensione coglie, in buona sostanza, l'opposizione fra moderatismo e radicalismo. Sul punto vedi Ricolfi (1994a e 1994b). 
TAB. 4. Insediamenti territoriali secondo la ricostruzione di Sani (1994)

\begin{tabular}{lcrrrr}
\hline & Padania & Etruria & Sud-1 & Sud-2 & Italia \\
\hline Dc & 26,6 & 21,7 & 35,5 & 47,2 & 31,0 \\
Pds-Rc & 17,0 & 39,9 & 19,5 & 16,1 & 22,2 \\
Psi & 12,3 & 12,6 & 15,1 & 15,2 & 13,5 \\
Leghe & 18,4 & 4,5 & 0,6 & 0,3 & 7,6 \\
Laici & 8,8 & 8,9 & 12,3 & 10,0 & 9,9 \\
Msi & 4,1 & 4,4 & 7,6 & 5,4 & 5,4 \\
Altri & 12,8 & 8,0 & 9,4 & 5,8 & 10,4 \\
\hline
\end{tabular}

TAB. 5. Come cambiano gli insediamenti territoriali

\begin{tabular}{llll}
\hline & 1992 & 1994 \\
\hline NORD & Lega & Lega & Forza Italia \\
& & Pds-Rc & \\
CENTRO & Pds-Rc & Forza Italia & \\
& & An & Pds-Rc \\
SUD & Dc-Psi & Forza Italia & \\
& &
\end{tabular}

attestano intorno al $15 \%$ in tutta Italia ${ }^{16}$. La destra ha vinto, e ha vinto soprattutto nel Nord, grazie alla contemporanea affermazione della Lega e di Forza Italia. Nelle regioni centrali continua l'egemonia dei partiti eredi del Pci, e Forza Italia si assesta su valori inferiori a quelli nazionali. Al Sud al posto della diarchia Dc-Psi subentra un cocktail instabile, formato da tre ingredienti eterogenei: Alleanza nazionale, il $\mathrm{Pds} \mathrm{e}$, in misura più contenuta, Forza Italia. Ora il governo centrale è soprattutto l'espressione delle preferenze elettorali del Nord (Tab. 5).

Quel che è sorprendente, tuttavia, è come la geografia del voto - ossia la partizione dell'Italia in zone elettoralmente omogenee - resti sostanzialmente immutata nonostante questi grandi cambiamenti. Una cluster sui risultati delle varie forze politiche presenti alle elezioni del 1994 ci ripropone, quasi esattamente, la

${ }^{16}$ In 22 circoscrizioni su 26 i risultati del Patto per l'Italia (Ppi + Patto Segni) sono compresi fra il $10 \%$ e il $20 \%$, e solo in Sardegna - grazie all'effetto Segni - superano il $25 \%$. 
partizione dell'Italia in tre zone che era emersa dal voto del 1992. Con un'importante qualificazione, però: la Sicilia costituisce un cluster a parte, una «quarta Italia» che si aggiunge - o meglio si «stacca» - dalle tre zone geopolitiche emerse dalle elezioni politiche precedent $\mathrm{i}^{17}$. Non solo, ma il voto siciliano è così eccentrico rispetto al voto meridionale che questa volta una eventuale rappresentazione semplificata, che si accontentasse di tre o addirittura due italie, non avrebbe l'effetto di riunificare i due Sud (come nella analisi di Sani delle elezioni del 1992), ma quello di accorpare Mezzogiorno e Regioni rosse ${ }^{18}$, lasciando la Sicilia isolata, oppure - a un livello di aggregazione ancora più spinto - quello di annettere la Sicilia alle regioni settentrionali.

Il diagramma ad albero mostra bene qual è la particolarità della nuova geografia del voto (Fig. 2): la Sicilia è più simile al Nord che al Mezzogiorno, e la vera frattura del paese non è fra il Mezzogiorno e il Centro-Nord, ma è fra il Centro-Sud (senza la Sicilia) e quello strano «can-gatto» ${ }^{19}$ costituito dal Nord più la Sicilia ${ }^{20}$ (Fig. 3).

17 La cluster è stata condotta con il metodo di Ward, sulla matrice delle distanze fra circoscrizioni elettorali derivante dalle percentuali di voto dei raggruppamenti seguenti: Pds+Rc, Rete+Verdi+Ad, Psi, Ppi+Patto Segni, Forza Italia+Lista Pannella+Ccd, Lega, An. Le aggregazioni sono state rese necessarie dall'assenza tecnica (mancanza di firme e vizi di forma) della maggior parte delle liste minori in numerose circoscrizioni. Solo nel caso della Lega la sua assenza nel Centro-sud è stata assunta come un dato politico (assenza di radicamento), e non come un fatto prevalentemente tecnico. Le circoscrizioni considerate sono 25, per l'esclusione della Valle d'Aosta (dove si votava solo con il sistema uninominale) e della Puglia (dove l'assenza tecnica di Forza Italia rende molto arduo qualsiasi confronto). I profili delle circoscrizioni sono stati calcolati escludendo i collegi in cui il peso delle «Altre liste» (diverse dalle 13 liste-base) superava il $15 \%$ dei voti validi; questi collegi sono 7 in tutto, di cui 6 in Trentino Alto Adige e 1 in Molise.

La soluzione prescelta (a 4 cluster) ha un rapporto fra varianza inter-classe e varianza totale pari al $60,3 \%$, il che le assicura il rispetto del criterio di separazione nella sua versione «debole» (Ricolfi 1989). Una ulteriore riprova della bontà della soluzione a 4 è fornita dall'analisi discriminante, che assicura il $100 \%$ di attribuzioni corrette.

${ }^{18}$ Le Marche, a seconda delle specifiche adottate nella cluster, vengono aggregate ora alle Regioni rosse ora al Mezzogiorno. Sul carattere intermedio del profilo elettorale delle Marche vedi, più avanti, il diagramma della fig. 4 .

${ }_{19}$ Che l'aggregazione Nord + Sicilia possa rivelarsi un «can-gatto» (Sartori 1991) si può intuire dal notevole guadagno di omogeneità che la soluzione a 4 cluster comporta rispetto a quelle a 2 e a 3 (vedi, più avanti, la nota 21).

${ }^{20}$ L'importanza relativa delle due fratture dipende, naturalmente, dagli indicatori che si utilizzano. Con dati elettorali la maggiore importanza della nuova frattura territoriale risulta nitidamente da tre circostanze:

1) partendo dalle 4 Italie da noi individuate la prima agglomerazione è fra Regioni rosse e Mezzogiorno, ossia fra due realtà profondamente diverse dal punto di vista della cultura civica; 
Né si sottovaluti la plausibilità di questa inedita aggregazione. Un'analisi dei parametri tecnici delle varie soluzioni mostra che una soluzione a due italie non è affatto implausibile, e che - se non si vogliono più di 5 Italie - la vera alternativa è fra una soluzione a due e una soluzione a quattro ${ }^{21}$.

2) la Sicilia non viene restituita al Mezzogiorno neppure nello stadio successivo, che ne vede invece l'annessione al Nord;

3) l'analisi discriminante associata alla cluster analysis mette in rilievo due funzioni discriminanti la più importante delle quali (in termini di varianza spiegata) rappresenta precisamente l'opposizione fra la diade Nord + Sicilia e la diade Regioni rosse + Mezzogiorno.

${ }^{21}$ Ecco la serie completa delle ultime 9 distanze di fusione:

\begin{tabular}{|c|c|c|c|c|}
\hline Passo & & distanza & variaz. & salti \\
\hline $10 \rightarrow 9$ & : & 627,9 & - & \multirow{7}{*}{6} \\
\hline $9 \rightarrow 8$ & $:$ & 757,4 & 129,5 & \\
\hline $8 \rightarrow 7$ & : & 905,5 & 148,1 & \\
\hline \multirow[t]{2}{*}{$7 \rightarrow 6$} & : & 1074,2 & 168,7 & \\
\hline & & & $\ldots$ & \\
\hline $6 \rightarrow 5$ & : & 1376,4 & 302,2 & \\
\hline \multirow[t]{2}{*}{$5 \rightarrow 4$} & : & 1740,0 & 363,6 & \\
\hline & & & $\ldots$ & \multirow[t]{3}{*}{4} \\
\hline $4 \rightarrow 3$ & : & 2480,9 & 740,9 & \\
\hline $3 \rightarrow 2$ & : & 3320,9 & 840,0 & \\
\hline & & & $\cdots .$. & \multirow[t]{2}{*}{2} \\
\hline $2 \rightarrow 1$ & : & 6568,2 & 3247,3 & \\
\hline
\end{tabular}

Come si vede la distanza di fusione, che può essere interpretata come il «prezzo» di una mancata agglomerazione, presenta gli incrementi più marcati passando da 6 a 5 cluster (conviene fermarsi a 6), da 4 a 3 cluster (conviene fermarsi a 4) e da 2 a 1 cluster (conviene fermarsi a 2). Questo significa che sia la soluzione a tre sia la soluzione a cinque sono cattivi compromessi fra parsimonia e omogeneità dei cluster. Nel caso la soluzione da noi suggerita (a 4 cluster) apparisse ancora troppo aggregata, occorrerebbe «saltare» a una soluzione a 6, con conseguente doppia scissione del Nord e del Mezzogiorno.

Il primo si scinderebbe secondo linee non troppo dissimili da quelle indicate da Diamanti nella sua recente analisi del voto leghista (Diamanti 1994, 59-61): da una parte tutto il Veneto, la circoscrizione Lombardia 2 (Varese, Como, Sondrio, Lecco, Bergamo, Brescia) e i collegi del Trentino Alto Adige sopravvissuti al nostro criterio sulla consistenza delle «Altre liste» (vedi nota 17), dall'altra tutte le altre circoscrizioni del Nord; la prima area corrisponde alle zone di origine del movimento leghista, la seconda alle aree più urbanizzate e/o di più recente insediamento della Lega, in cui oggi prevale Forza Italia.

Il secondo si scinderebbe in una parte settentrionale, comprendente tutto il Lazio, l'Abruzzo e la circoscrizione Campania 1 (che include Napoli) e in una parte meridionale comprendente tutte le altre circoscrizioni. Sul piano elettorale a dividere queste due aree è soprattutto la consistenza del Patto per l'Italia, che è debole nella prima e relativamente forte nella seconda.

Si noti la simmetria fra queste due partizioni del Nord e del Mezzogiono. Entrambe, infatti, non fanno che scorporare le aree più laiche e urbanizzate rispetto a 


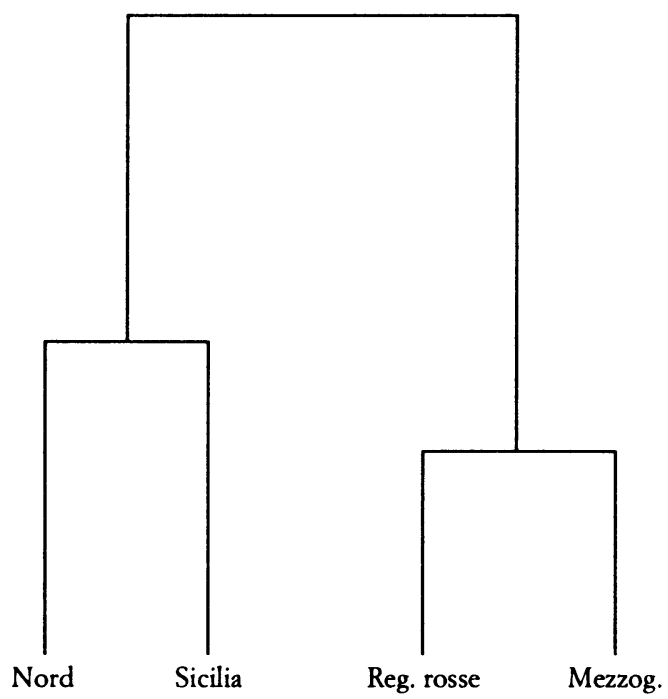

FIG. 2. Le quattro Italie (risultati della cluster)

In che cosa consiste l'anomalia del voto siciliano? Per capirlo conviene riportare i profili delle quattro Italie individuati con la cluster analysis ${ }^{22}$ (Tab. 6).

Come si vede le specificità del voto siciliano sono essenzialmente tre. La prima è la straordinaria affermazione di Forza Italia, che qui raggiunge il $33,3 \%$, contro il $21 \%$ nazionale e il $19,6 \%$ delle altre regioni meridionali. Questa affermazione è tanto più significativa se messa in relazione al peso assoluto e relativo di Alleanza nazionale, che in Sicilia rappresenta circa un quarto del cartello di destra, mentre nel resto del Mezzogiorno ne rappresenta circa la metà.

Il secondo aspetto è la debolezza delle due «chiese» che hanno fatto la storia della prima repubblica: il Ppi e i due partiti eredi del Pci (Pds e Rc). E vero che Rifondazione comunista non era presente, e che è quindi possibile che una parte dei

quelle più periferiche o tradizionali (il «profondo nord» leghista, e il «profondo sud» cattolico).

${ }^{22}$ Nel calcolo dei profili sono state escluse la Valle d'Aosta e la Puglia, ma sono stati inclusi i 7 collegi (del Trentino Alto Adige e del Molise) che nella cluster analysis erano stati esclusi a causa del peso eccessivo delle Altre liste. 


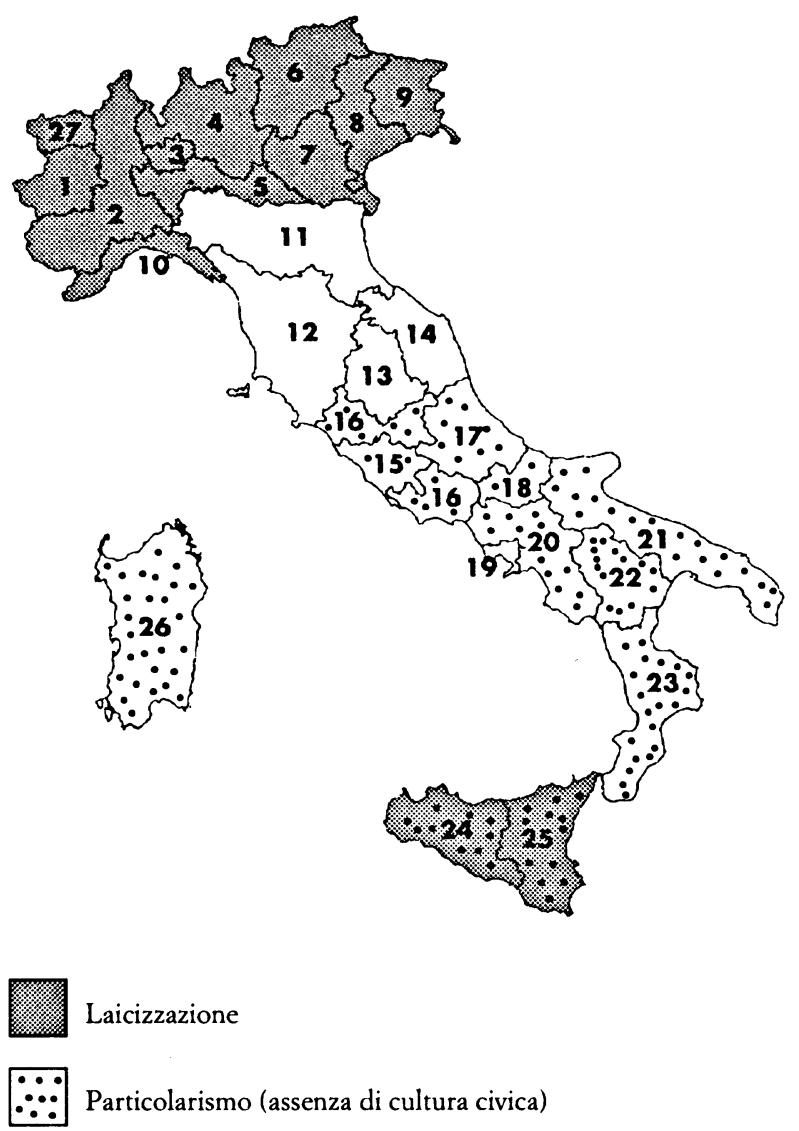

FIG. 3. Le 4 Italie

voti ad essa destinati siano confluiti nella Rete, ma anche tenendo conto (grazie al successivo risultato alle europee) di questa possibile fonte di distorsione, resta il fatto che la somma dei voti delle forze di più diretta ispirazione marxista (Pds e Rc) e cattolica (Ppi), che nelle altre zone del paese oscilla fra il $35 \%$ e il $60 \%$, qui raggiunge appena il $30 \%$.

Il terzo aspetto è la composizione della sinistra, in cui - caso unico in tutta Italia - le componenti laiche (Rete, Verdi, Ad e Psi), hanno il medesimo peso di quelle di ispirazione marxista (nelle altre zone del paese il rapporto è appena di 1 a 4 ). 
TAB. 6. Profili delle 4 Italie (senza la Puglia)

\begin{tabular}{lrcrr}
\hline & Nord & Reg. rosse & Mezzog. & Sicilia \\
\hline Pds+Rc & 19,2 & 42,7 & 28,3 & 16,7 \\
Verdi+Ad+Rete & 5,2 & 5,2 & 5,3 & 12,5 \\
Psi & 1,5 & 2,2 & 3,0 & 2,9 \\
Ppi+Patto & 15,4 & 14,8 & 16,4 & 13,9 \\
Pannella & 3,6 & 2,9 & 3,4 & 3,4 \\
Lega & 19,0 & 3,3 & 0,0 & 0,0 \\
Forza Italia & 24,8 & 16,8 & 19,6 & 33,3 \\
An & 7,4 & 11,4 & 20,4 & 13,9 \\
Altri & 3,9 & 0,7 & 3,6 & 3,4 \\
& & & & \\
Totale & 100,0 & 100,0 & 100,0 & 100,0 \\
\hline
\end{tabular}

Il voto di marzo e l'enigma siciliano

Prima di proseguire nell'analisi del voto, vorrei sottolineare che, nonostante il loro carattere controintuitivo, la soluzione a due e la soluzione a quattro Italie sono estremamente solide. I quattro bacini geografici individuati non sono costrutti arbitrari, comode semplificazioni di una realtà assai più sfumata. In questo caso - a differenza che in altri in cui io stesso mi sono imbattuto (Ricolfi 1989) - le quattro Italie non solo superano alcuni test fondamentali interni alla tecnica utilizzat ${ }^{23}$, ma sono nitidamente riconoscibili anche adottando tecniche molto diverse dalla cluster. Con un'analisi delle corrispondenze sulla tabella che incrocia le circoscrizioni e le liste elettorali ${ }^{24}$, ad esempio,

${ }^{23}$ Mi riferisco, in particolare, al test del diametro dei cluster: se la variabilità intraclasse è inferiore alla variabilità interclasse, allora il diametro medio dei cluster è inferiore alla distanza media fra i relativi baricentri (Ricolfi 1989). Nel nostro caso il rapporto fra variabilità intra (within) e inter (between) è pari a 2:3, il che corrisponde a una varianza spiegata del $60 \%$.

${ }^{24}$ L'analisi delle corrispondenze è stata condotta sulla tabella che incrocia le circoscrizioni elettorali con le quote di consenso dei partiti. Sono state considerate tutte le circoscrizioni eccetto Valle d'Aosta e Puglia. I partiti considerati sono Rc, Pds, Rete+Verdi+Ad, Psi, Ppi+Patto Segni, Lega, Forza Italia+Ccd, Alleanza nazionale, Pannella+Altri; quest'ultima aggregazione ha lo scopo di minimizzare la presenza di celle zero nella tabella di contingenza. Per lo stesso motivo i consensi di Rifondazione comunista in Sicilia sono stati stimati (redistribuendo il totale ottenuto da Rc, Pds e Rete alle Politiche in modo proporzionale ai voti ottenuti dai tre schieramenti alle Europee).

Il metodo di normalizzazione utilizzato è quello suggerito da Carroll, Green e Schaffer (1986), ora implementato in Spss come opzione di default (normalizzazione «canonica»). I primi due assi fattoriali rappresentano 1 ' $82,3 \%$ dell'inerzia totale. 
bastano le prime due coordinate $(82,3 \%$ dell'inerzia totale) per rendere perfettamente riconoscibili $\mathrm{i}$ quattro raggruppamenti (Fig. 4).

Solo due circoscrizioni - Marche e Campania 2 - occupano regioni dello spazio intermedie fra un cluster e l'altro. Per il resto i quattro raggruppamenti sono chiaramente individuabili ad occhio nudo.

Così con un'analisi discriminante ${ }^{25}$. Le prime due funzioni discriminanti $(93,0 \%$ della varianza totale) garantiscono il $100 \%$ di attribuzioni corrette, come si può anche vedere dall'elevato grado di separazione fra i cluster nel diagramma che rappresenta le coordinate delle circoscrizioni sulle prime due funzioni discriminanti (Fig. 5).

Dunque, per capire il voto di marzo dobbiamo capire il senso delle fratture che la nuova geografia del voto segnala. E per capire questo senso è essenziale riuscire a sciogliere l'enigma del voto siciliano. Sono convinto che se si è in grado di risolvere questo enigma, diventa anche più facile capire la nuova mappa geo-politica dell'Italia.

Vorrei essere molto esplicito su un punto. Il voto siciliano costituisce un fatto rispetto al quale non si presentano spiegazioni ovvie. Quel che va spiegato, infatti, non è perché la Sicilia ha scelto Berlusconi ma perché si è comportata in modo radicalmente difforme rispetto al resto del Mezzogiorno.

Proprio per questa intrinseca difficoltà del problema, dopo il 27 marzo ho interrogato moltissime persone - studiosi, giornalisti, militanti, osservatori politici - cercando di raccogliere congetture ed ipotesi. Fra queste spiegazioni vorrei ricordarne quattro:

a) l'influenza della mafia, vista come soggetto che è ancora in grado di controllare una parte considerevole dell'elettorato;

b) la diffidenza dei siciliani, supposta maggiore di quella degli altri elettorati meridionali e ritenuta incompatibile con la vaghezza delle promesse elettorali della sinistra ${ }^{26}$;

c) la delusione per i risultati della giunta Orlando, e l'insofferenza per le risse al «tavolo dei progressisti»;

${ }^{25}$ L'analisi discriminante è stata effettuata esattamente sugli stessi dati sottoposti a cluster analysis. La varianza delle prime due funzioni discriminanti è pari al $93,0 \%$ del totale; le tre correlazioni canoniche valgono $0,96,0,94$ e 0,78 .

${ }^{26}$ Questa ipotesi mi è stata prospettata da Diego Gambetta. 


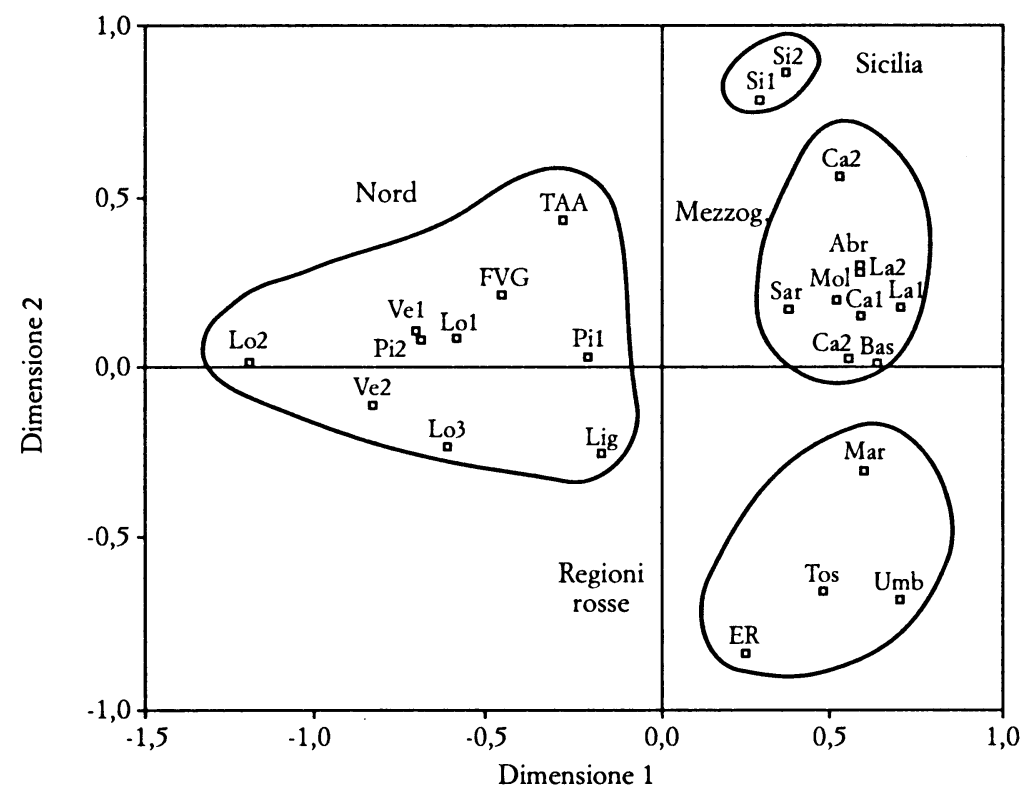

FIG. 4. Posizioni delle circoscrizioni nel piano fattoriale

d) l'instabilità dell'elettorato siciliano, sempre pronto a spostare grandi masse di voti da un'opzione all'altra.

$\grave{E}$ inutile soffermarsi sulla debolezza di queste spiegazioni. A parte l'ultima, che - in quanto capace di render conto di qualsiasi esito - è una non-spiegazione, è abbastanza evidente il loro carattere ad boc. La prima - influenza della mafia - presuppone, senza spiegarlo in modo convincente, un comportamento completamente difforme fra mafia da un lato, e camorra e 'ndrangheta dall'altro. La seconda - diffidenza dei siciliani - sembra sottovalutare l'entità del pronunciamento siciliano per Forza Italia o, simmetricamente, sembra postulare una radicale e indimostrata differenza psicologica dell'elettore siciliano. La terza - delusione per i progressisti e per la giunta Orlando - sembra dimenticare che il disgusto per le risse dei progressisti è stato universale, e soprattutto non dice come mai l'insoddisfazione per il sindaco sia confluita su Forza Italia anziché su Alleanza nazionale ${ }^{27}$.

${ }^{27}$ In Sicilia, a differenza che in altre parti del Mezzogiorno, il consenso al Msi-Al- 


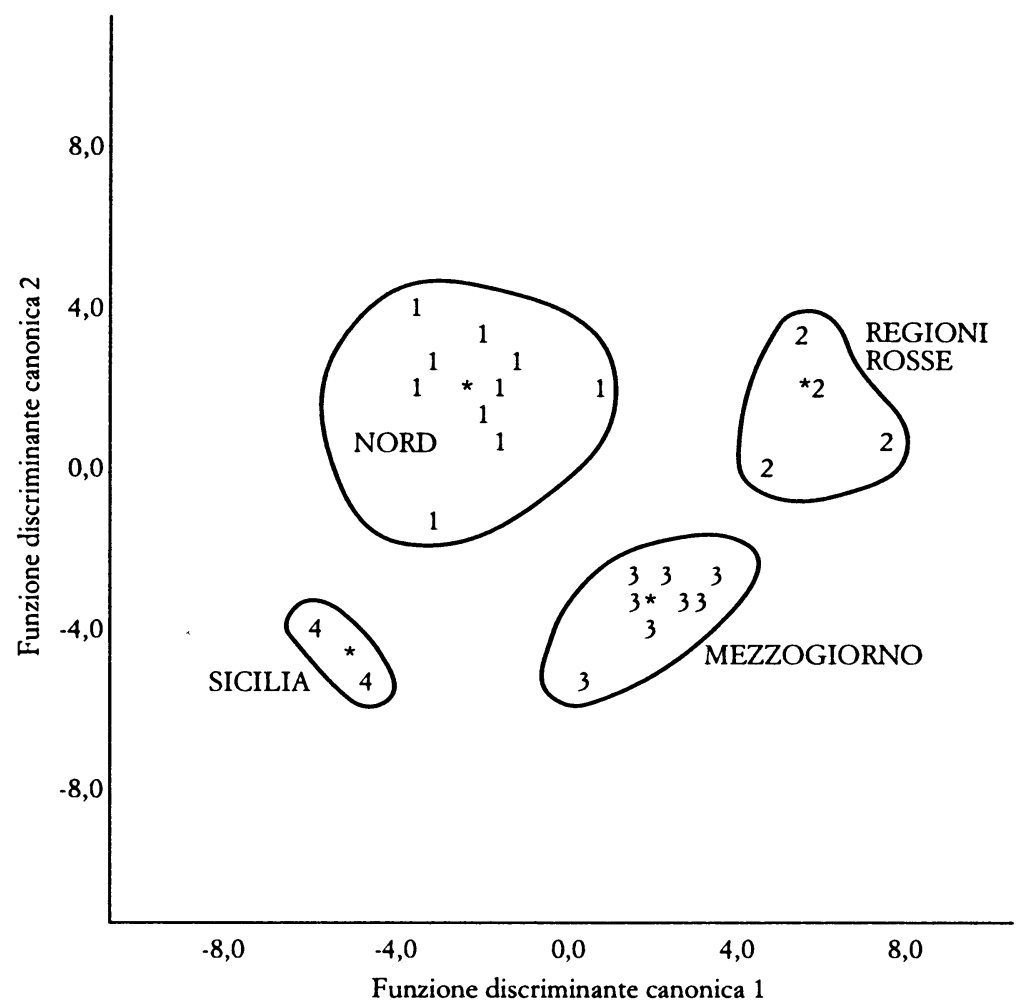

FIG. 5. Coordinate delle circoscrizioni sulle prime due funzioni discriminanti

A costo di essere un po' schematico, vorrei provare a suggerire una lettura alternativa di quel che è successo il 27 marzo. La mia interpretazione può essere condensata in questi sei semplici punti:

1. È almeno un decennio che il sistema politico italiano è entrato in una fase di laicizzazione accelerata.

2. Il grado di laicizzazione del voto è molto diverso nelle varie realtà regionali, e costituisce oggi una delle fratture fondamentali del nostro sistema politico. esploso, sfiorando il $35 \%$. 
3. Negli ultimi 4 anni, da quando la Lega è diventata un protagonista della politica nazionale ${ }^{28}$, l'alternativa centralismo/ federalismo (o statalismo/liberismo, come alcuni preferiscono chiamarla) è diventata una delle poste fondamentali dello scontro politico.

4. Il tratto fondamentamentale di Forza Italia è la sua laicità.

5. Il voto siciliano a Forza Italia è innanzitutto un voto laico e anti-centralista.

6. Se fra le regioni meridionali la Sicilia, e solo la Sicilia, ha potuto esprimere un voto di questo tipo è perché nella cultura dell'isola esiste un cocktail unico - ossia assente nelle altre regioni meridionali - fra laicizzazione della politica e istanze autonomiste.

In quel che segue cercherò di fornire qualche supporto empirico a questa interpretazione che, è bene sottolinearlo, allo stato attuale delle conoscenze disponibili resta una semplice congettura.

Laicizzazione della politica e crisi del centralismo: una nuova frattura territoriale

Per laicizzazione della politica intendo, essenzialmente, un aumento del voto di opinione a scapito del voto di appartenen$\mathrm{za}^{29}$. L'esistenza di un trend di questo genere non ha bisogno di essere provata, perché è stata documentata e sottolineata in molti lavori e in numerosissime circostanze. Qui vorrei ricordare soltanto che fin dall'indagine del 1983 il rapporto Iard segnalava proprio questa deriva nelle preferenze giovanili, ed ipotizzava che di lì a non molti anni tale deriva si sarebbe traslata sulle preferenze dell'intero corpo elettoral ${ }^{30}$. La crisi dei due «partiti-chiesa» - la Dc e il Pci - e l'ascesa dei partiti laici, soprattutto nuovi ( $\operatorname{Pr}$ e Verdi prima, Rete e Lega negli anni più recenti) è uno dei leit motiv di quasi tutte le ricerche sui giovani condotte a partire dagli anni ' 80 .

${ }^{28}$ Ricordiamo che ancora alle politiche del 1987, con un contingente di appena due parlamentari, la Lega era praticamente assente dalla scena politica nazionale. È solo fra il 1989 e il 1990 che, con i successi ottenuti alle elezioni europee ma soprattutto a quelle amministrative, la Lega diventa un protagonista non marginale della politica nazionale. In proposito si veda la puntuale ricostruzione di Diamanti (1993), in particolare il cap. II.

${ }^{29}$ La distinzione fra i tre tipi di voto si deve a Parisi e Pasquino (1977).

30 Vedi Ricolfi (1984, 86-91). 
Lo stesso discorso appena richiamato sulla laicizzazione della politica potrebbe essere ripetuto, mutatis mutandis, per la crisi del centralismo. Per tutti gli anni ' 70 e ' 80 , mentre i principali paesi dell'Occidente si impegnavano - talora anche con governi di sinistra - in politiche di stampo liberista (si pensi alle politiche di deregulation attuate dai conservatori negli Usa e in Gran Bretagna, ma anche alle privatizzazioni del governo socialista in Francia), l'Italia non modificava di una virgola l'indirizzo inflazionistico ed assistenziale della sua politica economica. Né la cosa può stupire più di tanto, se si pensa che in tutto il dopoguerra nessun partito italiano - e meno che mai il partito liberale dell'era Craxi - ha mai propugnato politiche di tipo liberi$\mathrm{sta}^{31}$. In queste condizioni la nascita di una forza come la Lega e la diffusione, anche al di fuori dell'area di destra, di una cultura del libero mercato (si pensi ad Ad, e allo stesso Patto Segni), è il minimo di reazione che ci si potesse attendere da un organismo gravemente malato come l'Italia della metà degli anni ' 80 .

Ora, il punto è che questi due processi non si sono limitati ad investire il nostro paese in modo più o meno graduale e uniforme, ma vi hanno attecchito in modo repentino e - soprattutto - territorialmente differenziato. Sulla grande frattura fra la «cultura civica» delle regioni del centro-nord e il «particolarismo» delle regioni meridionali (Putnam 1993, Cartocci 1994) una frattura il cui senso si potrà discutere all'infinito, ma della cui corposa presenza è davvero difficile dubitare - si è innestata una nuova e diversa frattura, quella fra le regioni centraliste e le regioni anticentraliste, fra le regioni più ideologizzate e le regioni politicamente più laiche, in cui il declino del voto di appartenenza è in uno stadio molto più avanzato.

Ma questa frattura - e qui sta il fatto nuovo delle elezioni del 27 marzo - non si sovrappone in alcun modo alla vecchia frattura fra Centro-Nord e Mezzogiorno, ma la taglia trasversalmente. Vento liberista e divorzio fra elettori e partiti-chiesa si alimentano a vicenda solo nel Nord. Il risultato è che le regioni rosse, nonostante la loro appartenenza all'Italia che sta al di sopra della fatidica linea Grosseto-Ascoli Piceno, finiscono per somigliare più alle regioni meridionali che a quelle del triangolo industriale, o alle altre regioni della terza Italia.

${ }^{31}$ Sull'assenza di un'autentica tradizione liberista nei partiti storici del nostro paese vedi Ricolfi (1993a, 61-4). 
D'altro canto il processo di laicizzazione della politica e l'ostilità verso lo stato centrale non sono appannaggio esclusivo del Nord. Anche la Sicilia, nonostante si collochi parecchio più a sud della linea che separa i «cives» dai «barbari», è attraversata da venti e da umori per molti versi simili a quelli del Nord. La Sicilia, insieme alla Sardegna, è l'unica regione meridionale ad avere una robusta tradizione autonomista e anti-centralista (si pensi all'esperienza milazzista ${ }^{32}$ alla fine degli anni '50). Da questo punto di vista le due isole sono le sole regioni del Mezzogiorno in cui il consenso a Forza Italia non deve «scontare» l'impopolarità connessa all'alleanza con la Lega nelle regioni «separatiste» e «antimeridionali» del Nord. Inoltre - come nel Nord e a differenza che in Sardegna - in Sicilia il peso dei partiti più centralisti e ideologici è minore che nel centro-sud. Se come indicatore (inverso) di laicizzazione utilizziamo il peso dei partiti di ispirazione marxista, cattolica e fascista otteniamo questo quadro:

TAв. 7. Peso dei partiti ideologici

\begin{tabular}{lccc}
\hline Circoscrizione & 1992 & 1994 & Variaz. \\
\hline Piemonte 1 & 45,1 & 45,0 & $-0,1$ \\
Piemonte 2 & 44,4 & 40,2 & $-4,2$ \\
Lombardia 1 & 43,0 & 39,7 & $-3,3$ \\
Lombardia 2 & 44,3 & 36,8 & $-7,5$ \\
Lombardia 3 & 50,1 & 44,5 & $-5,6$ \\
Trentino Alto Adige & 32,5 & 28,7 & $-3,8$ \\
Veneto 1 & 48,9 & 46,1 & $-2,8$ \\
Veneto 2 & 46,8 & 42,3 & $-4,4$ \\
Friuli Venezia Giulia & 50,6 & 47,6 & $-3,0$ \\
Liguria & 52,4 & 52,4 & 0,0 \\
Emilia Romagna & 61,4 & 66,4 & 5,0 \\
Toscana & 65,8 & 68,7 & 2,9 \\
Umbria & 71,6 & 76,9 & 5,3 \\
Marche & 69,3 & 70,5 & 1,2 \\
Lazio 1 & 62,1 & 68,8 & 6,7 \\
Lazio 2 & 68,4 & 65,2 & $-3,2$ \\
Abruzzo & 69,7 & 63,8 & $-5,9$ \\
Molise & 74,1 & 63,1 & $-11,0$ \\
Campania 1 & 63,5 & 62,4 & $-1,1$ \\
Campania 2 & 64,4 & 62,0 & $-2,4$ \\
Basilicata & 71,6 & 67,2 & $-4,4$ \\
Calabria & 64,1 & 67,4 & 3,2 \\
Sicilia 1 & 58,6 & 42,0 & $-16,6$ \\
Sicilia 2 & 61,6 & 47,0 & $-14,6$ \\
Sardegna & 59,9 & 64,8 & 4,9 \\
ITALIA & 56,5 & 54,4 & $-2,1$ \\
\hline
\end{tabular}

32 Sul milazzismo vedi, ad esempio, Battagli, D'Angelo e Fedele (1988) e Anastasi (1993). 
È interessante notare che mentre la spaccatura fra il Nord e il resto del paese era già evidente nel 1992, l'annessione della Sicilia al Nord - per quanto annunciata fin dal 1992 da un peso dei partiti ideologici relativamente ridotto rispetto a quello delle altre regioni del Centro-Sud - si consuma solo in quest'ultima tornata elettorale. Dal punto di vista del grado di laicizzazione della politica, la Sicilia è oggi indistinguibile dal Nord, così come il resto del Mezzogiorno è indistinguibile dalle regioni rosse.

Questo risultato può apparire sorprendente, ma non è certo isolato. La debolezza dei partiti ideologici non è l'unico indicatore che segnala una affinità fra le regioni del Nord e la nostra isola maggiore. Se, ad esempio, dalla laicizzazione della politica passiamo alla secolarizzazione in senso stretto, scopriamo che la distanza fra le regioni del Nord e la Sicilia è molto ridotta. Una recente mappa della religiosità elaborata da Cartocci per il 1991 mostra che in tutto il Nord solo 9 province su 33 sono altamente secolarizzate. Le altre si dividono più o meno equamente fra province bianche e province «in transizione». La Sicilia, d'altro canto, è l'unica regione del Mezzogiorno in cui le province a religiosità «conformista» non sono la totalità o la schiacciante maggioranza: tutte le province orientali (Messina, Catania, Siracusa) sono «in transizione», ossia ricadono nella medesima classe di Asti, Vercelli, Novara, Pavia, Torino, Trento, Bolzano, Pordenone e Udine ${ }^{33}$.

Ma persino sul piano della cultura civica, che indubbiamente rappresenta il terreno su cui la distanza fra il Centro-Nord e il Mezzogiorno (Sicilia inclusa) resta più elevata, l'eccentricità del caso siciliano appare in tutta evidenza. Uno studio di D'Amico e Raniolo mostra che in Sicilia fin dal 1987 l'astensionismo aggiuntivo, ossia il più tipico indicatore del voto di scam$\mathrm{bio}^{34}$, si è allineato sui valori dell'Italia nel suo insieme, e nel 1991 - in occasione del referendum sulla preferenza unica - è addirittura sceso al di sotto del valore medio nazionale.

Del resto la Sicilia e il Nord sono le uniche due aree del paese in cui, fin dalla metà degli anni '80, è comparsa un'oppo-

33 Vedi Cartocci (1994), pp. 178-186.

34 L'astensionismo aggiuntivo è «l'astensionismo per così dire specifico delle tornate referendarie, depurato cioè da quello espresso normalmente in altre consultazioni» (D'Amico, Raniolo 1994, 376). Sull'astensionismo aggiuntivo e sul suo uso come indicatore del voto di scambio si vedano Parisi (1974), Parisi e Rossi (1978), D'Amico (1982), Cartocci (1990). 
sizione radicale al sistema politico. Anzi, la Rete e la «primavera di Palermo» precedono di qualche anno le prime significative affermazioni elettorali della Lega ${ }^{35}$. Forse la «rivoluzione italiana» è anche il risultato di una improvvisa e paradossale sintonizzazione fra il neo-particolarismo della protesta anti-fiscale del Nord e l'uscita dal particolarismo di una parte non trascurabile della società civile siciliana.

Se si tiene conto di questa nuova frattura, della contrapposizione fra regioni centraliste e regioni autonomiste, la suddivisione dell'Italia in quattro zone suggerita dalla cluster analysis riceve un'interpretazione molto semplice e diretta.

Le quattro Italie altro non sono che il risultato dell'innesto, sulla tradizionale frattura fra il particolarismo del Sud e le culture civiche del Centro-Nord, della nuova frattura fra centralismo e autonomismo, statalismo e liberismo, voto di appartenenza e voto di opinione. Viste in questa prospettiva le regioni rosse e la Sicilia rappresentano i luoghi di rottura del nesso fra autonomismo e cultura civica ( $\mathrm{Tab}$. 8). Le regioni rosse sono quella parte del paese in cui il grado di laicizzazione della politica è basso rispetto alla forza della cultura civica, la Sicilia è quella parte del paese in cui il livello di cultura civica è basso rispetto al grado di laicizzazione della politica. Si potrebbe dire, usando la tipologia di Parisi e Pasquino, che nelle regioni del Nord prevale il voto di opinione, nelle regioni del Mezzogionno prevale il voto di scambio. Nelle regioni rosse al voto di opinione si affianca il voto di appartenenza, in Sicilia al voto di scambio si affianca il voto di opinione.

Questo schema molto semplice ma anche molto potente ${ }^{36}$ vale finché la base della classificazione sono le regioni o le cir-

35 Devo questa osservazione ad una conversazione con Rocco Sciarrone.

${ }^{36}$ È nota la capacità degli indicatori di cultura civica di discriminare fra CentroNord (poco voto di scambio) e Mezzogiorno (molto voto di scambio). Secondo Cartoc$\mathrm{ci}$, ad esempio, su base provinciale la varianza dell'astensionismo aggiuntivo spiegata da tale frattura territoriale si aggira intorno al $50 \%$, con un picco del $63 \%$ in corrispondenza del referendum del 1993 (Cartocci 1994, 39-41). Per avere un'idea comparativa del potere discriminante delle due fratture territoriali abbiamo ripetuto i calcoli di Cartocci su base circoscrizionale, confrontando la capacità della frattura tradizionale di spiegare le differenze di astensionismo aggiuntivo con la capacità della nuova frattura (Nord+Sicilia versus Regioni rosse+Mezzogiorno) di spiegare le differenze di consenso ai partiti ideologici (indicatore inverso di laicizzazione). Il risultato mostra che la nuova frattura è molto più discriminante della vecchia $(90,9 \%$ contro $66,3 \%)$. Sono grato a Cartocci per avermi messo a disposizione il file originario su cui ha effettuato i suoi calcoli. 
TAB. 8. Le due fratture territoriali

\begin{tabular}{lcc}
\hline & \multicolumn{2}{c}{ CULTURA CIVICA } \\
& forte & debole \\
\hline CENTRALISMO & Reg. rosse & Mezzogiorno \\
& Nord & Sicilia \\
\hline
\end{tabular}

coscrizioni, ma diventa assai meno nitido non appena si scende nel livello di aggregazione, anche solo a livello provinciale. Se fino al livello delle circoscrizioni la mappa dell'Italia è una mappa a quattro colori abbastanza regolare, non appena si scende di un gradino il quadro si fa molto più variegato. $\mathrm{Ci}$ sono province del Mezzogiorno (e non solo della Sicilia) in cui la politica si sta rapidamente laicizzando, e ci sono province del Centro-Nord in cui la cultura civica è relativamente debole. La mappa geo-politica dell'Italia somiglia a una carta geografica se le unità sono le regioni o le circoscrizioni, ma diventa il vestito di Arlecchino se le unità sono le province.

\begin{tabular}{lccc}
\hline & Scambio & Opinione & Appartenenza \\
\hline Nord & - & + & - \\
Regioni rosse & - & + & + \\
Mezzogiorno & + & - & + \\
Sicilia & + & + & - \\
\hline
\end{tabular}

\section{La geometria dello spazio elettorale}

In quel che precede abbiamo cercato di interpretare il senso delle quattro zone emerse dalla cluster ipotizzando l'esistenza di una nuova frattura territoriale, ancora più importante e profonda di quella che divide in due l'Italia sulla linea «che corre dalla foce della Fiora a quella del Tronto» (Cartocci 1994, 95).

Dell'esistenza di una simile frattura abbiamo fornito molti indizi, ma nessuna prova diretta. È vero, la dicotomia che oppone le regioni del Centro-Sud all'accoppiata «Nord più Sicilia» si lascia interpretare in termini di laicizzazione della politica. Ma questo non vuol dire che siano proprio la laicizzazione della politica e l'anti-centralismo le dimensioni lungo le quali le 
circoscrizioni elettorali si dividono fra loro. Nulla esclude che il senso di quella frattura sia un altro.

Per poter scegliere questa chiave di lettura a scapito di altre occorre chiedersi se la nostra interpretazione della frattura è compatibile con un'analisi della geometria dello spazio elettorale condotta allo stesso livello di aggregazione territoriale. Un'analisi del genere non dice, naturalmente, come gli individui percepiscono lo spazio elettorale ma quali sono le dimensioni che dividono fra loro gli elettorati delle circoscrizioni. Quel che un'analisi della geometria dello spazio elettorale condotta a livello macro (ecologico) ci può aiutare a scoprire, in altre parole, è il principio d'ordine che regola le differenze territoriali nel voto.

A questo scopo abbiamo condotto un'analisi fattoriale sulla matrice di correlazione fra $\mathrm{i}$ consensi a otto liste o raggruppamenti di liste:

\section{Rifondazione comunista \\ Pds \\ Rete+Verdi+Ad \\ Psi \\ Ppi+Patto Segni \\ Lega \\ Forza Italia + Pannella \\ Alleanza nazionale}

Le aggregazioni sono state rese necessarie dall'assenza «tecnica», per mancanza di firme, di tutte le liste minori (eccetto Psi e Rifondazione) in molte circoscrizioni. Dall'analisi è stata esclusa la Puglia per l'assenza di Forza Italia. In Sicilia, dove Rifondazione non era presente, le quote di consensi di Rc, Pds e Rete sono state ricostruite utilizzando le informazioni contenute nel voto europeo ${ }^{37}$. Con una rappresentazione bidimensionale si ottiene il diagramma della figura 6 .

Nel diagramma, la cui struttura generale non cambia scendendo nel livello di aggregazione territoriale fino al livello dei collegi, gli unici due assi chiaramente interpretabili sono proprio l'asse liberismo-statalismo (o autonomismo-centralismo) e l'asse di laicizzazione della politica.

37 Sul metodo di stima vedi la nota 24 . 


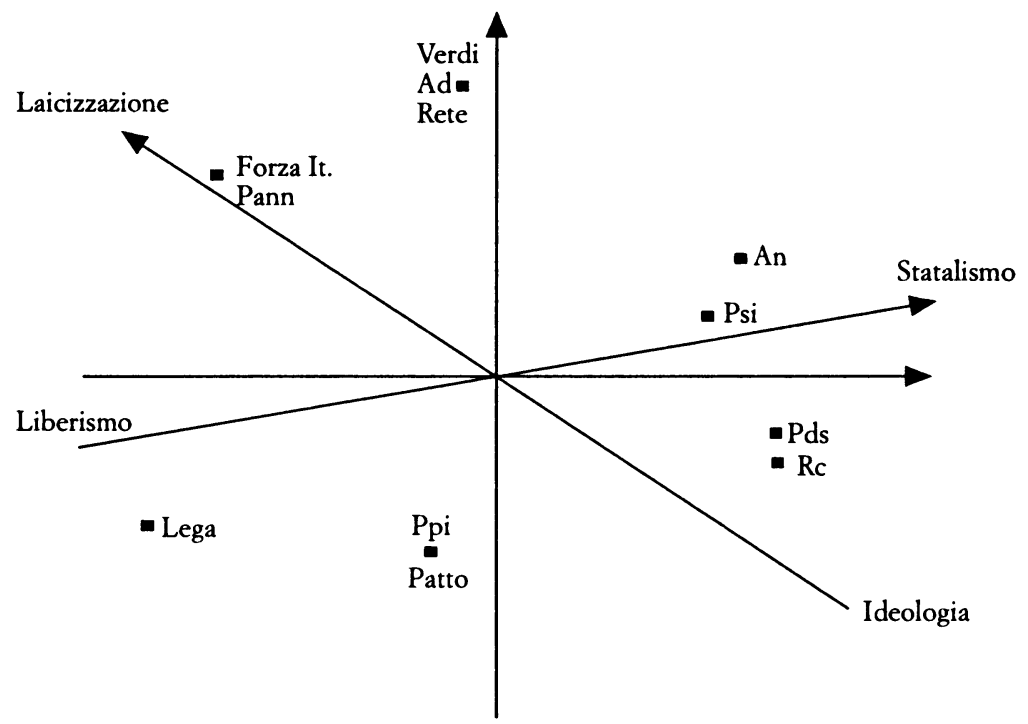

FIG. 6. Geometria dello spazio elettorale a livello macro

Il primo - liberismo-centralismo - oppone Lega e Forza Italia ai partiti tradizionalmente difensori dell'intervento dello stato nell'economia: Alleanza nazionale, che si rifà al corporativismo fascista e alla cosiddetta «economia sociale di mercato», i due partiti eredi del Pci (Pds e Rc), e infine il Psi che - per quanto molto trasformato negli ultimi vent'anni - è sempre rimasto fedele ad una concezione attiva (per non dire altro) del ruolo dello stato nell'economia.

Il secondo asse - laicizzazione della politica, o voto di opinione versus voto di appartenenza - oppone Forza Italia ed alleati laici del Pds a tutti i partiti di matrice ideologica. Comunismo (Rc, Pds), fascismo (An), cattolicesimo (Ppi e Patto) e socialismo (Psi) si ritrovano accomunati al polo negativo dell'asse di laicizzazione. In questo caso è la Lega a trovarsi nel mezzo, come è logico tenuto conto dello status ambivalente della Lega come partito. La Lega è un partito non ideologico, organizzato come un partito di massa (Biorcio 1991).

Può stupire che un partito come il Psi, che da tempo non ha più né un'organizzazione da partito di massa né una precisa identità ideologica, occupi un punto dello spazio elettorale molto prossimo a quello di Alleanza nazionale, e venga quindi - 
nella nostra ricostruzione - annesso al gruppo dei partiti ideologici. In realtà questa attribuzione è del tutto logica. L'analisi della posizione del Psi è effettuata nel 1994, ossia dopo la «cura dimagrante» che gli elettori hanno riservato al partito post-craxiano delle segreterie Benvenuto e Del Turco. Paradossalmente, proprio l'azione di rinnovamento e di moralizzazione intrapresa dai due segretari-sindacalisti ha avuto l'effetto di appannare, agli occhi degli elettori, i tratti più moderni e «disinvolti» dell'immagine del partito, a tutto vantaggio di quelli più tradizionali e quindi anche più ideologici. Il nuovo, nel caso del Psi, come in quello della Dc, ha dovuto assumere le sembianze di un «ritorno alle origini». È molto probabile che nell'esigua pattuglia che oggi vota ancora Psi $\mathrm{i}$ «puri» e i nostalgici prevalgano nettamente sui «rampanti» e sui clienti.

Se questa interpretazione dell'analisi fattoriale ha qualche fondamento, diventa legittima anche un'altra lettura del relativo diagramma. Tracciando gli assi fattoriali l'uno in modo da isolare i partiti ideologici, l'altro in modo da isolare i partiti di massa, il quadro che si ottiene si può leggere come una vera e propria tipologia dei partiti italiani.

Questa tipologia ha un certo interesse perché mostra che oggi, sul piano territoriale, la consueta opposizione fra destra e sinistra, ma anche quella più recente fra vecchio e nuovo, sono più utilmente sostituite da due dicotomie che colgono il tipo di rapporto che lega partiti ed elettori ${ }^{38}$. Se si vuole capire la distri-

TAB. 9. Tipologia dei partiti

\begin{tabular}{|c|c|c|c|}
\hline & & \multicolumn{2}{|c|}{ ORGANIZZ. DI MASSA } \\
\hline \multirow{3}{*}{ 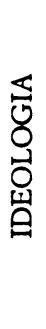 } & & sì & no \\
\hline & sì & $\begin{array}{l}\text { Pds } \\
\text { Rc } \\
\text { Ppi }\end{array}$ & $\begin{array}{l}\text { An } \\
\text { Psi }\end{array}$ \\
\hline & no & Lega & $\begin{array}{l}\text { Forza It. } \\
\text { Pann. } \\
\text { Ad } \\
\text { Verdi } \\
\text { Rete }\end{array}$ \\
\hline
\end{tabular}

38 Sull'emergenza, nel corso della XI Legislatura, dell'opposizione fra vecchio e nuovo come dicotomia che integra la tradizionale dicotomia destra-sinistra, vedi Ricolfi (1993b, 1994a, 1994b). 
buzione territoriale del voto la doppia distinzione evidenziata dalla tipologia è uno strumento più efficace di quelli consueti. Non solo, ma la tipologia dei partiti ha una precisa corrispondenza con la quadripartizione dell'Italia. Le quattro celle della tabella 9, infatti, non individuano solo altrettante forme-partito $\mathrm{ma}$ anche altrettanti insediamenti territoriali. Se ad ogni gruppo di partiti facciamo corrispondere l'area del paese in cui il suo seguito elettorale è più elevato, quel che ritroviamo è precisamente la partizione dell'Italia da cui la nostra analisi ha preso le mosse (Tab. 10). Limitando l'analisi ai quattro partiti maggiori otteniamo:

TAB. 10. Corrispondenze fra zone geopolitiche e forme-partito

\begin{tabular}{|c|c|c|c|}
\hline & & \multicolumn{2}{|c|}{ ORGANIZZ. DI MASSA } \\
\hline \multirow{5}{*}{ 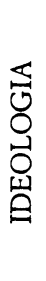 } & & sì & no \\
\hline & \multirow{2}{*}{ sì } & Pds & An \\
\hline & & REG. ROSSE & MEZZOGIORNO \\
\hline & \multirow{2}{*}{ no } & Lega & Forza It. \\
\hline & & NORD & SICILIA \\
\hline
\end{tabular}

Tutto ciò sembra indicare che le quattro Italie rappresentano anche altrettante forme di organizzazione e canalizzazione del consenso. La distinzione fra Centro-Nord e Sud non coglie solo la presenza o meno della cultura civica, ma anche il grado di radicamento degli attori politici - i partiti di massa - che ne sono stati il principale veicolo. Così l'opposizione fra il CentroSud e la diade Nord+Sicilia non si limita a cogliere l'opposizione fra due politiche - l'una centralista, l'altra anti-centralista ma riflette anche la capacità degli attori politici di mettersi in sintonia con quel processo di laicizzazione della politica che nell'elettorato italiano è in corso da almeno un decennio.

\section{Sulla natura di Forza Italia}

E siamo arrivati, finalmente, al punto cruciale del nostro ragionamento. Qual è la natura di Forza Italia? 
Come in quasi tutte le cose, anche sul carattere del consenso a Forza Italia e sulle ragioni del suo successo ci sono almeno due «scuole di pensiero». La prima la si potrebbe chiamare la scuola del re-labeling. Secondo questo modo di vedere - che proprio nell'interpretazione del voto siciliano ha avuto modo di esprimersi nel modo più esplicito - con il passaggio dalla prima alla seconda Repubblica tutto quel che è cambiato sono le etichette dei partiti. Prima c'erano la Dc e il Psi, ora ci sono Forza Italia e Alleanza nazionale. Le vittorie dei progressisti alle amministrative erano pura apparenza. Un'analisi differenziale dei consensi ottenuti dalle persone dei sindaci e dalle liste che li sostenevano basta a mostrare la fragilità e l'illusorietà delle grandi vittorie dei progressisti. Nel voto politico di marzo l'elettorato non ha cambiato preferenze, semplicemente ha convogliato i voti del vecchio quadripartito sulle forze che meglio garantivano la continuità fra vecchio e nuovo regime (Blando e Comito 1994).

L'altra lettura è diametralmente opposta. Forza Italia ha sfondato non perché era in grado di riciclare il vecchio, ma perché meglio dei progressisti ha saputo interpretare la voglia di cambiamento degli italiani. Che poi questa voglia di cambiamento sia interpretata da alcuni come richiesta di de-regulation, da altri come bisogno di un leader, da altri ancora come domanda di ricambio del ceto politico poco importa. Il punto centrale è e resta il legame fra successo di Forza Italia e richiesta di innovazione del sistema politico.

Il modo più semplice per vagliare queste due interpretazioni è quello di partire da un'analisi attenta della provenienza dei consensi a Forza Italia. Usando il panel Cra possiamo individuare facilmente la provenienza degli elettori di Forza Italia, che qui e nelle tavole successive - per ragioni di omogeneità dei confronti - abbiamo preferito aggregare agli alleati del Centro cristiano democratico (presente solo in Molise) e della lista Pannella (presente in 18 circoscrizioni su 26).

Cominciamo con le preferenze elettorali 10 mesi prima del voto (maggio 1993), calcolate per l'Italia nel suo insieme, al netto della Puglia (in cui mancava la lista di Forza Italia) e della Sicilia (dove mancava la lista di Rifondazione comunista) (Tab. 11).

I risultati non potrebbero essere più sorprendenti. Contro il cliché che vede Forza Italia come un partito nettamente spostato a destra, scopriamo invece che quasi il 30\% dell'elettorato di 
TAB. 11. Composizione dell'elettorato di Forza Italia (con e senza lista Pannella)

\begin{tabular}{lccl}
\hline Liste & Con Pann. & Senza Pann. & Schieramenti \\
\hline Pds+Rc & 7,7 & 8,7 & Opposizione \\
Rete+Verdi+Pri & 21,5 & 16,9 & di sinistra \\
Pann & 10,2 & 8,2 & \\
Psi+Psdi+Pli & 15,3 & 17,2 & Pannella \\
Dc & 16,1 & 19,2 & e \\
Lega & 26,9 & 27,5 & quadripartito \\
Msi & 2,3 & 2,1 & Opposizione \\
\hline
\end{tabular}

Forza Italia proviene da sinistra. Si potrebbe supporre che l'ampiezza del contingente elettorale fornito dalla sinistra sia dovuta all'inclusione dei repubblicani nella sinistra, e della Lista Pannella nel cartello di Forza Italia. Ma non è così. Se scorporiamo i repubblicani, il contributo della sinistra scende di soli due punti, mentre per quanto riguarda l'impatto della Lista Pannella basta dare un'occhiata alla seconda colonna della tabella, che ripete il calcolo sui soli elettori di Forza Italia ${ }^{39}$, per rendersi conto che l'apporto della sinistra resta comunque superiore al $25 \%$.

Semmai, quel che si potrebbe obiettare è che, nel maggio del 1993, l'elettorato della Lista Pannella faceva prevalente riferimento alla sinistra, e andrebbe quindi, almeno in parte, aggiunto al «conto» che i progressisti hanno dovuto pagare al Cavaliere. In tal caso il contributo dell'area progressista a Forza Italia si porterebbe intorno al $35 \%$, ossia non molto al di sotto del peso che quest'area aveva nella primavera del 1993 (Tab. 1). E come dire che voto a Forza Italia e «matrice di sinistra» sono quasi stocasticamente indipendenti: chi è di sinistra non ha una propensione a scegliere Forza Italia significativamente inferiore a quella dell'elettorato nel suo insieme!

Per salvare l'idea che Forza Italia non abbia nulla a che fare con la sinistra possiamo anche, naturalmente, immaginare che l'elettorato di sinistra confluito in Forza Italia sia costituito da persone che, nel frattempo, hanno cambiato orientamento politico. Chi crede alla teoria del «vento di destra» può pensare che quella fetta di consensi che Forza Italia ha drenato a sinistra fossero, comunque, destinati a finire a destra. Sfortunatamente, Molise.

${ }^{39}$ Inclusi i 2.500 voti circa ottenuti dal Ccd (Centro cristiano democratico) in 
ancora una volta i dati Cra sono lì a smentirlo. Una settimana prima del voto, ai membri del panel Cra era stato chiesto, oltre a quale fosse la loro intenzione di voto il 27 marzo, anche qual era la loro seconda scelta. Ebbene, su 100 elettori intenzionati a votare Forza Italia quasi 20 , come seconda scelta, indicavano un partito di sinistra.

$\mathrm{Ma}$ le sorprese non finiscono qui. Chi pensa che Forza Italia non sia altro che la Dc «riverniciata» deve constatare che nonostante la forza di attrazione degli ex democristiani del Ccd - nel cartello di Forza Italia il peso dell'elettorato della Dc è addirittura inferiore, sia pur di poco, al peso della Dc a metà del 1993. Né si può dire che Forza Italia sia, in buona sostanza, il risultato di un'abile manovra di recupero e riconversione dell'elettorato del vecchio quadripartito. Il peso dell'elettorato di quadripartito in Forza Italia è valutabile intorno al 30-35\%, appena al di sopra del consenso che il quadripartito stesso conservava nella primavera del 1993.

Resta, infine, da valutare il nesso fra Forza Italia e le opposizioni di destra. Ma anche qui la relazione è debolissima: gli elettori provenienti da Lega e Movimento sociale rappresentano il $28-29 \%$ della base di Forza Italia, contro un peso della destra a maggio del 1993 valutabile intorno al $25-26 \%$.

Questi risultati sono del tutto incompatibili con la lettura «continuista», che vede Forza Italia come la reincarnazione della Dc o del quadripartito. Ma, a ben vedere, creano qualche problema anche alla lettura che sottolinea gli elementi di rottura con il passato, la capacità del partito di Berlusconi di presentarsi come l'alfiere del nuovo. Per rendersi conto di questo punto conviene calcolare, per ogni insieme di liste, la sua propensione a votare Forza Italia (Tab. 12).

Dopo l'elettorato radicale, che ha una propensione molto alta a votare il cartello Forza Italia per l'ovvia circostanza che la Lista Pannella ne fa parte, i due elettorati più attratti da Forza Italia sono quelli della Lega e degli alleati laici della Dc: Psi, Psdi e Pli. Se per il primo (elettorato della Lega) l'interpretazione «nuovista» può anche funzionare, ed è anzi rafforzata dalla relativamente alta propensione a votare Forza Italia dei «partiti nuovi» alleati del Pds (Verdi e Rete), per il secondo non funziona affatto. Il terzetto Psi-Psdi-Pli, che ha una propensione altissima a votare Forza Italia, è costituito dai tre partiti più coinvolti in Tangentopoli (Ricolfi 1993c). Come conciliare questa circostanza con l'idea che Forza Italia sia l'espressione della vo- 
TAB. 12. Propensione a votare Forza Italia (con e senza lista Pannella)

\begin{tabular}{lccl}
\hline Liste & Con Pann. & Senza Pann. & Schieramenti \\
\hline Pds+Rc & 9,3 & 8,7 & Opposizione \\
Rete+Verdi+Pri & 24,4 & 16,9 & di sinistra \\
Pann & 43,0 & 8,2 & Pannella \\
Psi+Psdi+Pli & 39,0 & 17,2 & e \\
Dc & 20,4 & 19,2 & quadripartito \\
Lega & 34,0 & 27,5 & Opposizione \\
Msi & 9,8 & 2,1 & di destra \\
\hline
\end{tabular}

lontà degli elettori di lasciarsi alle spalle i mali della prima Repubblica?

Il fatto è che la tabella precedente ci consegna un paradosso. Forza Italia attira soprattutto gli elettorati che esprimono il massimo e il minimo di coinvolgimento con la prima Repubblica. Da un lato tutti i partiti nuovi, che non esistevano prima degli anni '70 (Lega, Rete, Verdi, Lista Pannella), dall'altro tre partiti (Psi, Psdi, Pli) il cui coinvolgimento negli scandali della prima Repubblica è maggiore di quello della stessa Dc.

E molto probabile che la ragione di questo apparente paradosso stia in una percezione «bifronte» della collocazione di Forza Italia su quella che, nel corso della XI Legislatura, è diventata una dimensione fondamentale dello spazio politico: l'asse di innovazione. Si può ipotizzare, in altre parole, che mentre per tutti gli altri partiti la loro posizione sull'asse di innovazione sia percepita abbastanza uniformemente da tutto l'elettorato, nel caso di Forza Italia questa percezione diventi bimodale. Gli elettori dei partiti «normali» si distribuiscono «a gobba di dromedario» (Fig. 7a), l'elettorato di Forza Italia si distribuisce «a gobba di cammello» (Fig. 7b).

Una parte degli elettori di Forza Italia dà poca importanza ai legami di Berlusconi con Craxi e con il vecchio regime, e ne dà molta al fatto che Forza Italia è la formazione politica più nuova, una formazione - tra l'altro - che ha il vantaggio di non presentarsi come un partito ma come un «movimento» o un insieme di club. Questi elettori tendono ad assegnare a Forza Italia un punteggio molto alto sull'asse di innovazione (gobba destra).

Un'altra parte dell'elettorato riconosce in Berlusconi, nel suo ottimismo e nella sua esaltazione della logica dell'impresa, 
la riedizione aggionata del dinamismo dell'era Craxi. Questi elettori tendono a vedere Forza Italia più come la promessa di un ritorno ai fasti degli anni ' 80 che come un mezzo per liberarsi definitivamente del retaggio di quell'epoca. È molto probabile che, per costoro, Forza Italia sia soprattutto un'assicurazione contro il «lato inquietante» del rinnovamento: il rallentamento dell'attività economica trainata dalle commesse pubbliche (si pensi alla legge Merloni subito sospesa da Berlusconi), il rischio di uno sconfinamento di Tangentopoli nelle aree finora poco battute della piccola corruzione e soprattutto dell'evasione fiscale (inchiesta sulla guardia di finanza). Questi elettori, in cuor loro, tendono a percepire Forza Italia nel registro della continuità $^{40}$ (gobba sinistra).

In realtà, l'unico elemento in grado di unificare $\mathrm{i}$ dati che siamo venuti esponendo è la distinzione fra partiti ideologici e partiti laici. Per rendersene conto basta riorganizzare la tabella precedente in modo da mettere in luce, simultaneamente, la provenienza politica e la provenienza culturale dei consensi a Forza Italia. Lasciando da parte la lista Pannella, che dà adito a più problemi interpretativi di quanti ne chiarisca, il quadro che si ottiene è il seguente (Tab. 13).

Si vede bene che mentre la distinzione fra sinistra e destra spiega solo una piccola parte della variabilità del consenso a Forza Italia, la distinzione fra partiti ideologici e partiti «laici» si rivela invece estremamente potente. In ciascuna delle tre aree politiche considerate l'attrazione che Forza Italia esercita nei confronti delle forze laiche è molto superiore a quella che esercita nei confronti dei partiti ideologici. Nella sinistra la propensione di Rete, Verdi e repubblicani a votare Forza Italia è quasi il triplo di quella dei partiti eredi del Pci $(24,4$ contro 9,3). Nell'area di quadripartito Psi, Psdi e Pli hanno una propensione doppia rispetto a quella della Dc $(39,0$ contro 20,4$)$. Nella destra, infine, i leghisti mostrano una propensione che è quasi il quadruplo di quella dei missini $(34,0$ contro 9,8$)$.

Prima di essere di destra o di sinistra, vecchio o nuovo, il

${ }^{40} \mathrm{Ci}$ si può fare un'idea della presenza e dell'ampiezza di questa componente di Forza Italia riflettendo sullo scarto fra le dimensioni dell'area moderata - pari al 35 . $40 \%$, secondo una stima recente (Ricolfi 1994b) - e le dimensioni del consenso che gli elettori hanno fornito al centro cattolico $(15,8 \%)$. Tutto lascia supporre che una frazione consistente di tale scarto sia andata ad ingrossare la «gobba sinistra» (percezione «continuista») dell'elettorato di Forza Italia. 


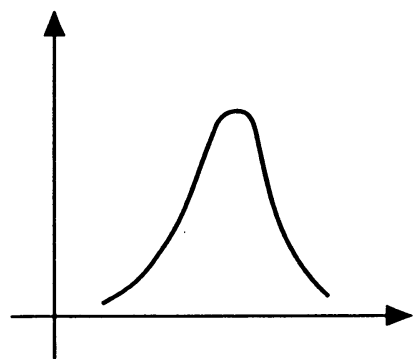

(a)

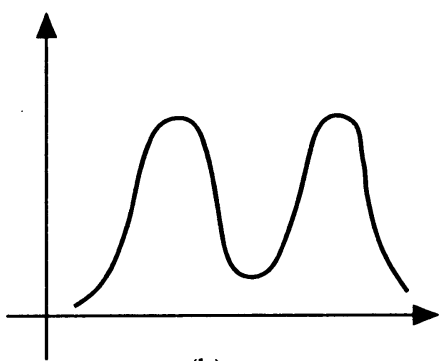

(b)

FIG. 7. Distribuzioni ipotetiche sull'asse di innovazione: elettorati-dromedario (a) ed elettorati-cammello (b)

TAB. 13. Propensione a votare Forza Italia

\begin{tabular}{llll}
\hline & Oppos. sin. & Quadripartito & Oppos. des. \\
\hline \multirow{2}{*}{ Ideologici } & Pds+Rc & Dc & Msi \\
& 9,3 & 20,4 & 9,8 \\
Non ideol. & Rete+Verdi+Pri & Psi+Psdi+Pli & Lega \\
& 24,4 & 39,0 & 34,0 \\
\hline
\end{tabular}

voto a Forza Italia è l'espressione - e in parte il veicolo - di quel processo di laicizzazione della politica da cui la nostra interpretazione aveva preso le mosse.

E ora?

Se il voto a Forza Italia, pur con tutte le sue sfaccettature motivazionali e territoriali ${ }^{41}$, è - prima di tutto - un voto anti-

${ }^{41}$ Certo, i nostri stessi dati, se analizzati con maggiore dettaglio - distinguendo fra il Nord e il Sud del paese, o considerando separatamente le varie forze politiche - sono in grado di segnalare anche tutta la complessità e le sfaccettature di un voto che non si lascia ricondurre ad una matrice univoca. Molti indizi, ad esempio, lasciano pensare che il carattere laico del voto a Forza Italia sia più accentuato nel Nord del paese, e che nelle regioni meridionali abbiano giocato un ruolo più importante componenti come l'inquietudine di una parte dell'elettorato di sinistra (Rifondazione e Verdi), e la capacità del vecchio personale democristiano riciclato nel $\mathrm{Ccd}$ di attirare una parte dell'elettorato che prima votava Dc. Voglio dire, con questo, che le due interpretazioni da cui abbiamo preso le mosse - quella del re-labeling e quella della vittoria del «nuovo» - pur 
ideologico, il voto siciliano cessa di costituire un enigma. La società siciliana era già, nel 1992, la più laica e secolarizzata del Mezzogiorno. Inoltre, insieme alla Sardegna, era l'unica regione meridionale non priva di tradizioni autonomiste. Chi pensa che sia stata soprattutto la mafia a dirottare i voti su Forza Italia - oltre a sopravvalutare il potere di controllo diretto della mafia, e a non spiegarci perché ciò che è riuscito alla mafia non sia riuscito alla 'ndrangheta e alla camorra - trascura un aspetto essenziale della situazione siciliana. In Sicilia la mafia non aveva bisogno di convincere la popolazione a votare Forza Italia per il semplice motivo che l'elettorato siciliano era già pronto a farlo per ragioni proprie, alcune antiche, come la tradizione anti-centralista, altre più recenti, come l'impetuoso processo di laicizzazione della politica. Si può pensare, naturalmente, che un maggiore grado di autonomia dal governo centrale piaccia alla mafia, o addirittura sia la condizione per riacquistare quel controllo incontrastato del territorio che l'azione recente dello Stato sembra averle sottratto. Questo però non significa che la mafia è la causa del successo di Forza Italia ma, tutt'al più, che esiste una sintonia fra interessi mafiosi e preferenze elettorali dei siciliani.

Chi nel voto siciliano vede solo l'affermazione di Forza Italia non coglie la logica interna, la «coerenza» si sarebbe tentati di dire, di un voto che - su tutti i fronti - ha espresso innanzitutto il commiato dell'elettore dell'isola dai partiti ideologici. In nessuna regione il peso, nell'ambito della sinistra, dei partiti di ispirazione marxista è basso come in Sicilia, e questo indipendentemente dal fatto che il calcolo venga effettuato sulle elezioni politiche (in cui mancava Rifondazione comunista) o sulle successive elezioni europee in cui Rifondazione era presente. In nessuna circoscrizione (eccetto il Molise) il tracollo del centro cattolico è più netto, e in pochissime circoscrizioni il peso del partito popolare è così basso rispetto a quello del Patto Segnit2. In nessuna regione del Mezzogiorno, infine, il peso di Alleanza nazionale rispetto all'insieme della destra è basso come in Sicilia.

essendo reciprocamente incompatibili, e in aperto conflitto con la maggior parte dell'evidenza empirica disponibile, riacquistano una moderata capacità interpretativa se applicate a realtà territoriali diverse. La teoria del re-labeling coglie alcuni aspetti del voto meridionale, mentre la visione «nuovista» è compatibile con alcuni aspetti del voto delle regioni centro-settentrionali.

${ }_{42}$ Il confronto, naturalmente, è stato effettuato per le sole circoscrizioni in cui erano presenti sia il Partito popolare sia il Patto Segni. 
La laicizzazione della politica non fornisce solo una chiave di lettura per leggere il successo di Forza Italia. Essa mette anche a nudo i limiti della sinistra. Si è molto discusso, dopo il voto, sulle ragioni della sconfitta dei progressisti, sulla sua evitabilità $o$ inevitabilità. La nostra analisi porta a spostare l'attenzione su un altro piano.

La sconfitta dei progressisti non solo era evitabile, ma era sulla «carta» di un'analisi delle preferenze elettorali ${ }^{43}$ - l'esito meno probabile delle elezioni di marzo. La sinistra ha fatto tutto il possibile per perderle, dilapidando in un tempo rapidissimo un vantaggio che - appena pochi mesi prima - appariva incolmabile. L'impresa era molto difficile, ma grazie alla bagarre delle candidature e grazie alla rinuncia a scegliere un leader, alla fine ci è riuscita. Il punto, però, non è che la sinistra ha perso queste elezioni, ma che queste elezioni erano le ultime che la sinistra poteva vincere. D'ora in poi il gioco cambia. Non tanto per effetto delle nuove regole elettorali, quanto perché è l'elettorato italiano ad essere cambiato. La debolezza del centro e della sinistra non sta tanto nei loro programmi, o nella loro immagine - tutte cose che si possono cambiare abbastanza facilmente - quanto nella loro cultura, o meglio nel tasso di ideologia che la loro cultura continua a veicolare. Nella destra sono i partiti ideologici ad essere in minoranza, nel centro e nella sinistra accade esattamente il contrario. Mentre nel cartello di destra è il peso di An a essere trascurabile rispetto al baricentro laico costituito da Forza Italia e dalla Lega, nella sinistra sono i «cespugli» laici (Rete, Verdi, Ad, Psi) ad essere trascurabili rispetto alla massa elettorale dei due partiti ex-comunisti. Lo stesso discorso vale per il Centro. Il Patto Segni rappresenta meno di un terzo della galassia cattolica, e - dentro il Patto stesso - le componenti laiche (Amato, La Malfa, Zanone) sono semplici «fili d'erba» del cespuglio Segni.

In queste condizioni il futuro della destra e il futuro della sinistra si presentano in termini alquanto asimmetrici. La destra

${ }^{43}$ Per analisi delle preferenze elettorali intendo un'analisi della domanda politica, che cerca di distinguere fra la collocazione dei «consumatori» (elettori) e la collocazione dei «marchi» (partiti) nello spazio elettorale (Ricolfi 1994b). In termini sostantivi mi riferisco al fatto che alle politiche del 1994 la struttura dell'offerta era molto diversa a sinistra e a destra: a sinistra non esistevano «attrattori» laici (forze politiche nuove, non ideologiche e non trascurabili nelle dimensioni), a destra ne esistevano ben due (Lega e Forza Italia). 
è già avviata sulla strada della de-ideologizzazione, e può permettersi di puntare sul partito unico perché il suo baricentro è Forza Italia, ossia il più laico dei partiti della destra. L'unificazione della destra intorno a Forza Italia non va contro la deriva delle preferenze elettorali, e può diventare difficile o controproducente solo per effetto di errori politici grossolani, come quello che Berlusconi ha commesso con il decreto «salva-ladri».

La sinistra, con la promozione di Massimo D'Alema al vertice del Pds e con le aperture del neo-segretario al Partito popolare, sembra anch'essa puntare verso un'unificazione del fronte progressista, realizzata intorno al Pds e aperta ai «valori» del socialismo e del cattolicesimo sociale. Il guaio (per la sinistra stessa) di una prospettiva del genere è che essa corre in una direzione che è diametralmente opposta a quella lungo la quale evolvono le preferenze elettorali degli italiani. Mentre l'elettorato italiano si sta laicizzando, e le elezioni del 1994 mettono sotto gli occhi di tutti la grande frattura territoriale fra le regioni in cui sopravvivono i partiti ideologici e quelle in cui il loro declino è andato molto avanti, il più grande partito della sinistra elegge un segretario che è soprattutto espressione delle seconde e che, forse proprio per questo, non appena eletto ripropone la «santa alleanza» fra gli ultimi partiti-chiesa rimasti sulla scena. Questa scelta, se la nostra analisi precedente è fondata, può solo avere l'effetto di mettere definitivamente fuori gioco la sinistra. Il partito unico dei progressiti, infatti, in una situazione in cui le forze laiche non hanno saputo dar vita a una formazione autonoma e di forza comparabile rispetto al Pds, non si presenterebbe come una grande forza laica nella cui orbita gravitano gli eredi delle grandi ideologie del passato ma, tutto al contrario, come un partito ideologico che - modernizzandosi - è riuscito ad annettersi vari tipi di cespugli e zolle.

In questa scelta, $O$ in questa «attrazione fatale» ${ }^{44}$, vi è natu-

44 L'«attrazione fatale» fra partito cattolico e Partito democratico della sinistra è ben esemplificata da alcuni brani di questa intervista, rilasciata da Rocco Buttiglione pochi giorni prima di essere eletto segretario del Partito popolare:

«D.: Perché D'Alema le piace e Veltroni no?

R.: $\mathrm{Ci}$ sono due vie d'uscita dal marxismo: una che fa perno sull'idea di una sinistra borghese individualistica e anarchicheggiante e che mi sembra disastrosa. L'altra è il ritorno alle radici etiche e cristiane del movimento operaio che la enciclica Laborem exercens descrive come protesta etica contro una situazione di ingiustizia e di danno. E allora si viene a formare un partito della giustizia sociale in condizioni di mercato.

D.: D'Alema secondo lei interpreta questa seconda via d'uscita?

$\mathrm{R}$ : D'Alema è stato comunista, vede i limiti del comunismo e si domanda cosa 
ralmente una logica, e probabilmente anche qualcosa di nobile, se non di eroico. La logica sta, semplicemente, nel fatto che il $\mathrm{Pds}$ fa $\mathrm{i}$ conti con quel che c'è, e non può inventare o creare dal nulla gli interlocutori di cui avrebbe bisogno; la mancanza dalla scena di un partito di «sinistra liberale» non è imputabile al Pds ma - semmai - alla fragilità della cultura laica in Italia e alla pochezza e litigiosità dei piccoli partiti (e dei piccoli uomini) che in questi anni hanno provato ad occupare questa regione dello spazio politico. L'eroismo sta nel coraggio di andare contro corrente, di opporre un mondo di valori a un mondo che marcia esattamente nella direzione opposta.

E tuttavia è difficile non vedere l'esito fatale che questi processi e queste scelte sembrano annunciare per la sinistra. Mentre per la destra l'alternativa fra concentrazione e pluralismo è un'opzione politica come le altre, per la sinistra - se il processo di laicizzazione della politica continuerà - l'alternativa non è fra concentrazione e pluralismo, ma è fra pluralismo e declino.

Partiti con l'idea di garantire il bipolarismo e l'alternanza, potremmo ritrovarci così, fra qualche anno, con il paradosso di un sistema che resta tripolare nelle preferenze dell'elettorato ${ }^{45}$, ma è sostanzialmente bipartitico nelle sue espressioni parlamentari. Con un'aggravante, però. Che, dopo essere stati i campioni del «pluralismo polarizzato» e del «bipartitismo imperfetto», ora rischiamo di diventare il primo paese del mondo che sperimenta il «bipartitismo senza alternanza».

c'era di buono nell'esperienza comunista. Io dico che Dio ha tanto amato l'Italia che anche una cosa intrinsecamente perversa come il comunismo da noi ha prodotto molti frutti buoni. Io sono nato a Gallipoli, nel sud. I cafoni meridionali prima chinavano la schiena sulle zolle per dieci anni, l'undicesimo avevano un momento di ribellione animalesca: bruciavano il municipio e ammazzavano qualche possidente, poi arrivava la truppa che ne uccideva qualcuno e loro tornavano a piegare la schiena per altri dieci anni. Il Partito comunista, non da solo ma più degli altri, gli ha insegnato a organizzarsi, a fare i sindacati, a scioperare quando era il caso, a contrattare, a votare, a difendere i propri diritti come cittadini. Questo mi sembra il filone positivo da continuare» (intervista rilasciata a Gianluca Luzi, in «La Repubblica», 26/7/1994).

45 Il carattere intrinsecamente tripolare dello spazio elettorale considerato a livello macro risulta con evidenza da un'analisi delle preferenze elettorali (Ricolfi 1994a). Sulla natura dei tre poli nel corso della XI legislatura vedi Ricolfi (1994b). 
Riferimenti bibliografici

Amaturo, E. e G. Ragone (1979), Elementi di sociologia economica, Napoli, Liguori.

Anasatasi, A. (1993), Il voto siciliano nel lungo andare, in M. Morisi (a cura di), Far politica in Sicilia, Milano, Feltrinelli.

Battagli, R., M. D’Angelo e S. Fedele (a cura di), (1988), Il Milazzismo. La Sicilia nella crisi del centrismo, Roma e Reggio Calabria, Gangemi.

Bianco, M.L. e L. Ricolfi (1993), Famiglie, individui, donne: vecchi e nuovi problemi negli studi di mobilità sociale, in $\mathrm{M}$. Palumbo (a cura di), Classi, disuguaglianze e povertà, Milano, Franco Angeli.

Biorcio, R. (1991), La Lega come attore politico: dal federalismo al populismo regionalista, in $\mathrm{R}$. Mannheimer (a cura di), La Lega Lombarda, Milano, Feltrinelli.

Blando, A. e S. Comito (1994) I progressisti in Sicilia: false vittorie e reali sconfitte, in «Segno», 153-4 (marzo-aprile), pp. 97-110.

Cartocci, R. (1987), Otto risposte a un problema: la divisione dell'Italia in zone politicamente omogenee, in «Polis», I, pp. 481-514.

- (1990), Elettori in Italia, Bologna, il Mulino.

- (1994), Fra Lega e Chiesa, Bologna, il Mulino.

Carroll, J., P.E. Green e C.M. Schaffer (1986), Interpoint Distance Comparisons in Correspondence Analysis, in «Journal of Marketing Research», 23, pp. 271-80.

Corbetta, P. (1993), La Lega e lo sfaldamento del sistema, in «Polis», VII, pp. 229-52.

Corbetta, P., A.M. Parisi e H.M.A. Schadee (1988), Elezioni in Italia, Bologna, Il Mulino.

D'Amico, R. e F. Raniolo (1994), L'elettorato siciliano e il referendum sulla preferenza unica del 1991, in M. Caciagli e P.V. Uleri, Democrazie e referendum, Bari, Laterza.

D'Amico, R. (1982), Una modalità negativa del 'voto di scambio': l'astensionismo in Sicilia, in «Quaderni dell'Osservatorio elettorale», 9, pp. 143-78.

Diamanti, I. e R. Mannheimer (a cura di) (1994), Milano a Roma, Roma, Donzelli.

Diamanti, I. (1993), La Lega. Geografia, storia e sociologia di un nuovo soggetto politico, Roma, Donzelli.

- (1994), La Lega, in I. Diamanti e R. Mannheimer (a cura di), Milano a Roma, Roma, Donzelli, 53-61.

Duesenberry, J.S. (1949), Income, Saving and the Theory of Consumers's Behaviour, Cambridge (Mass.), Harvard University Press, trad. it. Reddito, risparmio e teoria del comportamento del consumatore, Milano, Etas Kompass, 1969.

Elster, J. (1983), Sour Grapes, Cambridge, Cambridge University Press, trad. it. Uva acerba, Milano, Feltrinelli, 1989. 
Glass, D. (1954), Social Mobility in Britain, London, Routledge \& Kegan Paul.

Hagger, P., A. Cliffe e Frey, A. (1977), Locational Models, London, Edward Arnold Ltd.

Leibenstein, H. (1950), Band-wagon, Snob and Veblen Effect in the Theory of Consumer Demand, in «Quarterly Journal of Economics», 64 .

- (1976), Beyond Economic Man. A New Foundation for Microeconomics, Cambridge (Mass.), Harvard University Press.

Mannheimer R. e G. Sani (1987), Il mercato elettorale, Bologna, il Mulino.

Natale, P. (1994), La nuova mappa geopolitica, in I. Diamanti e R. Mannheimer (a cura di), Milano a Roma, Roma, Donzelli.

Parisi, A. (1974), Questione cattolica e referendum: l'inizio di una fine, in «il Mulino», XXIII, pp. 410-38.

Parisi, A. e G. Pasquino (1977), Relazioni partiti-elettori e tipi di voto, in A. Parisi e G. Pasquino (a cura di), Continuità e mutamento elettorale in Italia, Bologna, il Mulino, pp. 215-39.

Paris, A. e M. Rossi (1978), La relazione partiti elettori: quale lezione, in «il Mulino», pp. 503-48.

Putnam, R. (1993), Making Democracy Work. Civic Traditions in Modern Italy, Princeton, Princeton University Press, trad. it. La tradizione civica nelle regioni italiane, Milano, Mondadori, 1993.

Ricolfi, L. (1984), Associazionismo e partecipazione politica, in A. Cavalli et al., Giovani oggi, Bologna, il Mulino.

- (1989), Sul mito delle K Italie. Una critica delle tipologie degli italiani, in «Polis», III, pp. 445-78.

- (1990), I meccanismi della felicità e dell' infelicità, in «Rassegna Italiana di Sociologia», 4.

- (1993a), Politica senza fede: l'estremismo di centro dei piccoli leghi$s t i$, in «il Mulino», 1993, 1, pp. 53-69.

- (1993b), La geometria dello spazio elettorale in Italia, in «Rivista Italiana di Scienza Politica», XXIII, pp. 433-74.

- (1993c), L'ultimo parlamento, Roma, La Nuova Italia Scientifica.

- (1994a), Politica a quante dimensioni, in «il Mulino», 1, pp. 29-43.

- (1994b), Left, Voice E God. Riflessioni sulla geometria del mercato elettorale, in «Micro \& Macro Marketing», 1, pp. 93-111.

Rogoff, N. (1953), Recent Trends in Occupational Mobility, New York, Free Press.

Sani, G. (1993a), Le Italie del 5 aprile, in «Polis», VII, pp. 207-28.

- (1993b), 1992: la destrutturazione del mercato elettorale, in «Rivista Italiana di Scienza Politica», XXIII, pp. 539-65.

- (1994), Il verdetto del 1992, in R. Mannheimer e G. Sani (a cura di), La rivoluzione elettorale, Milano, Anabasi.

Sartori, G. (1991), Comparazione e metodo comparato, in G. Sartori e L. Morlino (a cura di), La comparazione nelle scienze sociali, Bologna, il Mulino. 